\title{
Neonatal NET-inhibitory factor and related peptides inhibit neutrophil extracellular trap formation
}

\author{
Christian C. Yost, ${ }^{1,2}$ Hansjörg Schwertz, ${ }^{2,3}$ Mark J. Cody, ${ }^{1,2}$ Jared A. Wallace, ${ }^{4}$ Robert A. Campbell, ${ }^{2,5}$ Adriana Vieira-de-Abreu, ${ }^{2,5}$ \\ Claudia V. Araujo, ${ }^{2,5}$ Sebastian Schubert, ${ }^{2,5,6}$ Estelle S. Harris, ${ }^{2,5}$ Jesse W. Rowley, ${ }^{2,5}$ Matthew T. Rondina, ${ }^{2,5}$ James M. Fulcher, ${ }^{7}$ \\ Curry L. Koening, ${ }^{5}$ Andrew S. Weyrich, ${ }^{2,5}$ and Guy A. Zimmerman ${ }^{2,5}$
}

1Department of Pediatrics/Neonatology, ${ }^{2}$ Program in Molecular Medicine, ${ }^{3}$ Division of Vascular Surgery, ${ }^{4}$ Department of Pathology, and ${ }^{5}$ Department of Internal Medicine, University of Utah, Salt Lake City, Utah, USA. ' $C$ Center for Thrombosis and Hemostasis, University of Mainz, Mainz, Germany. 'Department of Biochemistry, University of Utah, Salt Lake City, Utah, USA.

\begin{abstract}
Neutrophil granulocytes, also called polymorphonuclear leukocytes (PMNs), extrude molecular lattices of decondensed chromatin studded with histones, granule enzymes, and antimicrobial peptides that are referred to as neutrophil extracellular traps (NETs). NETs capture and contain bacteria, viruses, and other pathogens. Nevertheless, experimental evidence indicates that NETs also cause inflammatory vascular and tissue damage, suggesting that identifying pathways that inhibit NET formation may have therapeutic implications. Here, we determined that neonatal NET-inhibitory factor (nNIF) is an inhibitor of NET formation in umbilical cord blood. In human neonatal and adult neutrophils, nNIF inhibits key terminal events in NET formation, including peptidyl arginine deiminase 4 (PAD4) activity, neutrophil nuclear histone citrullination, and nuclear decondensation. We also identified additional nNIF-related peptides (NRPs) that inhibit NET formation. nNIFs and NRPs blocked NET formation induced by pathogens, microbial toxins, and pharmacologic agonists in vitro and in mouse models of infection and systemic inflammation, and they improved mortality in murine models of systemic inflammation, which are associated with NET-induced collateral tissue injury. The identification of NRPs as neutrophil modulators that selectively interrupt NET generation at critical steps suggests their potential as therapeutic agents. Furthermore, our results indicate that nNIF may be an important regulator of NET formation in fetal and neonatal inflammation.
\end{abstract}

\section{Introduction}

Formation of neutrophil extracellular traps (NETs) may be an important component in the defensive armamentarium of neutrophils (polymorphonuclear leukocytes [PMNs]) that allows them to capture, immobilize, and putatively kill microbes in the extracellular space (1-4). NET formation occurs by a novel cell death process often called NETosis, although "vital" NETosis, in which the neutrophils do not immediately die, has also been described $(3,5)$. The molecular mechanisms leading to NET formation have not been completely dissected and may depend in part on the stimulus $(1-3,6)$. Nevertheless, decondensation of chromatin and extrusion of DNA together with histones and granule contents, are central events $(1-3,5,6)$. Deimination of histones mediated by peptidyl arginine deiminase 4 (PAD4) (7-9) is thought to be a sine qua non for nuclear decondensation and NET formation (1).

NET-mediated capture and elimination of pathogens may complement traditional PMN antimicrobial activities including phagocytosis and intracellular killing $(2,10)$. Clinical observations indicate that defects in NET formation contribute to intractable infections in some instances $(2,11)$, but the importance of NETs in pathogen killing in vivo remains unclear and controversial (1-3). Conversely, there is substantial evidence that NETs and NETassociated factors, including histones and granule proteases, mediate vascular and tissue injury and that NET-mediated injury

Conflict of interest: The authors have declared that no conflict of interest exists. Submitted: July 22, 2015; Accepted: July 28, 2016.

Reference information: J Clin Invest. 2016;126(10):3783-3798. doi:10.1172/JCI83873. is a previously unrecognized mechanism of innate immune collateral damage to the host $(1-3,9,12)$. Experimental models and limited clinical observations suggest that intra- or extravascular NET formation contributes to tissue injury in bacteremia $(9,13$, 14), transfusion-related acute lung injury (15), primary graft dysfunction after lung transplantation (16), sterile vasculopathies and immune inflammation $(17,18)$, thrombosis (19), and influenza (20). Thus, NET formation may be an important maladaptive activity of neutrophils (1) if it is triggered inappropriately or is unregulated in infection and inflammation.

Human neonates have unique and complicated immune regulation, susceptibility to infection, and inflammatory pathology. Although the infant is in a sterile environment in utero, it can be challenged by pathogens and their products before or during labor (21). Furthermore, newborns are rapidly colonized with bacteria after delivery, a process associated with increases in circulating and bone marrow neutrophils (22-24). Complex adaptations appear to have evolved that prevent excessive, injurious inflammation in the perinatal period and in the abrupt neonatal transition from the protected intrauterine environment to continuous microbial colonization and exposure (25-27). These adaptations may, however, be accompanied by increased susceptibility to infection $(26,27)$. Earlier, we found that PMNs isolated from umbilical cord blood of preterm and term infants do not form NETs when stimulated and have a defect in NET-mediated bacterial killing, suggesting such an adaptation (28). Other investigators subsequently reported temporally delayed NET formation when isolated neonatal neutrophils were stimulated in vitro (29). In exploring the 


\section{A Preterm neonatal PMNs}

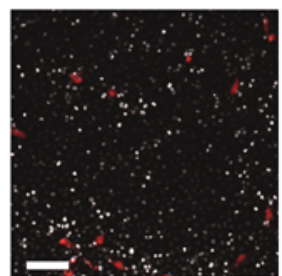

Day 0

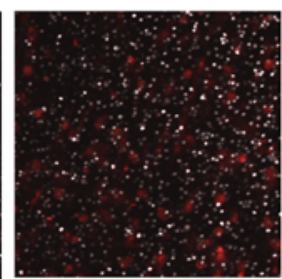

Day 3

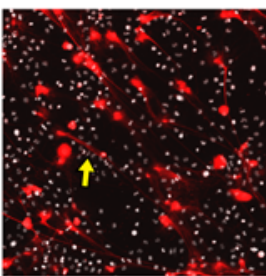

Day 14

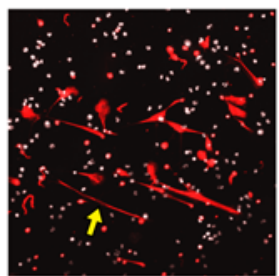

Day 28

\section{Maternal PMNs}

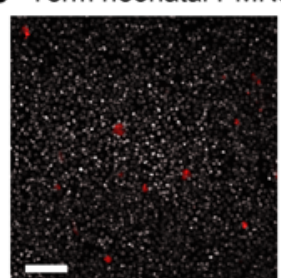

Day 0

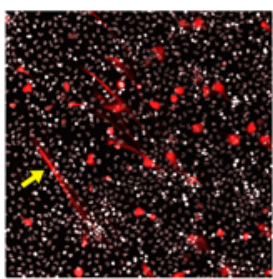

Day 2

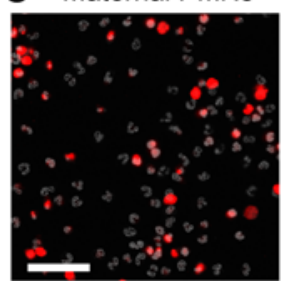

Control

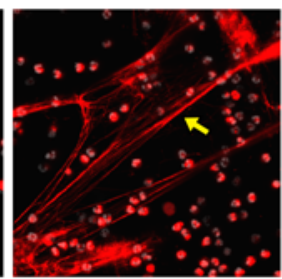

LPS
LPS

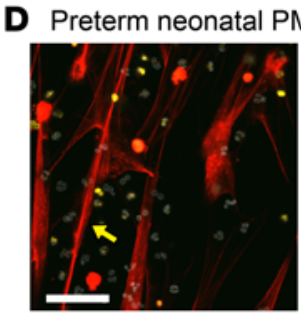

60 day plasma

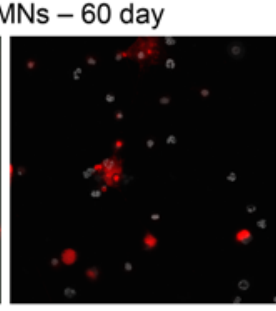

Cord blood plasma LPS

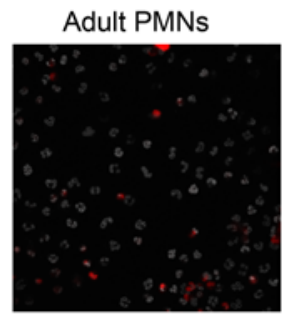

Cord blood plasma

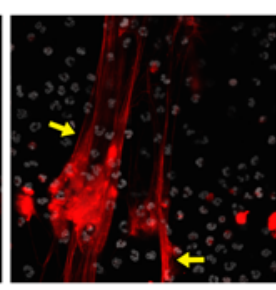

Autologous plasma LPS

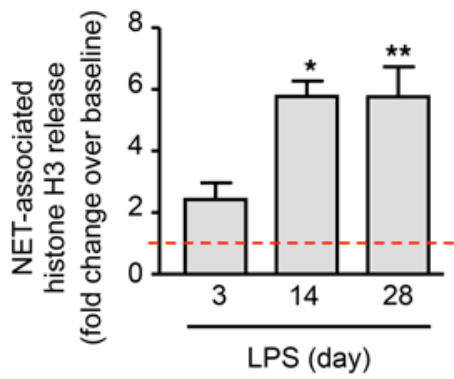

LPS (day)

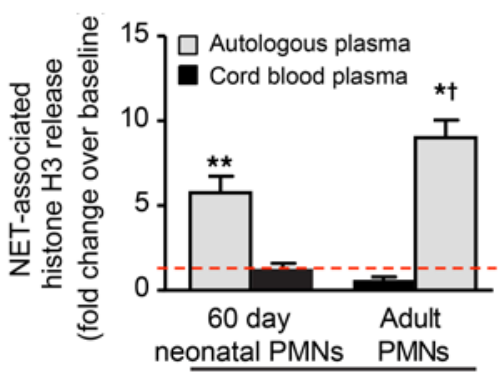

LPS

Figure 1. A NET- inhibitory factor is present in human umbilical cord blood. (A) Neutrophils from 7 preterm neonates were longitudinally examined over the first 28 days after birth for NET formation in response to LPS $(100 \mathrm{ng} / \mathrm{ml}, 1$ hour) assessed by live cell imaging (NETs, red fluorescence, yellow arrows; nuclear DNA, gray) and release of NET-associated histone H3 (fold change over baseline; mean \pm SEM). Original magnification, $\times 20$. Scale bar: $100 \mu \mathrm{m}$. One-way ANOVA with Tukey's post hoc testing. ${ }^{*} P<0.05 ;{ }^{* *} P<0.01$, compared with control histone $\mathrm{H} 3$ release arbitrarily set at 1 (red dashed line). (B) Neutrophils isolated from cord blood of a healthy term neonate on the day of delivery (left panel) or from venous blood on day 2 after birth (right panel) were stimulated with LPS (100 ng/ml, 1 hour) and imaged as in A. Analysis of NET formation by neutrophils from a second-term neonate yielded the same pattern. (C) Neutrophils isolated from venous blood of a healthy pregnant woman on the day of delivery were incubated in medium alone or stimulated with LPS $(100 \mathrm{ng} / \mathrm{ml})$ for 1 hour and imaged as in A. Neutrophils from a second healthy term mother also robustly formed NETs in response to LPS. Original magnification, $\times 60$. Scale bar: $100 \mu \mathrm{m}$. (D) Neutrophils were isolated from venous blood of 60 -day-old preterm neonates $(n=5)$, preincubated for 1 hour with day-60 autologous plasma or with stored autologous cord blood plasma, stimulated with LPS, and assessed for NET formation as in A. Original magnification, $\times 60$. Scale bar: $100 \mu \mathrm{m}$. Neutrophils isolated from venous blood of healthy adults and preincubated in autologous or stored cord blood plasma were studied in parallel. One-way ANOVA with Tukey's post hoc testing. ${ }^{*} P<0.05$, LPS/adult vs. LPS/neonatal; ${ }^{* *} P<0.01$, neonatal PMNs in autologous plasma vs. cord blood plasma; ${ }^{\dagger} P<0.001$, adult $P M N s$ in autologous plasma vs. cord blood plasma.

mechanism(s) for blunted neonatal NET deployment, we discovered a peptide in umbilical cord blood that inhibits NET formation in vitro and in vivo and that appears to be an endogenous regulator of NET generation. We also identified related peptides that inhibit NETosis. These previously unrecognized modulators of NET formation may have potential as selective antiinflammatory agents in addition to regulatory activities in specific inflammatory settings or tissue compartments.

\section{Results}

NET formation by human neonatal neutrophils is regulated by a peptide in umbilical cord blood. We examined in vitro NET deployment by neutrophils from umbilical cord blood on the day of delivery and from peripheral blood of infants collected at later days of life. NET formation was assessed qualitatively using live cell imaging with SYTO Green (cell permeable) and SYTOX Orange (cell impermeable) DNA stains and quantitatively by supernatant NET-associated histone $\mathrm{H} 3$ measurement (30). PMNs isolated from cord blood (day 0), whether from preterm $(n=8)$ or healthy term infants $(n=2)$, did not form NETs when stimulated (Figure 1, A and B), consistent with our earlier observations (28). Nevertheless, term and preterm neonates rapidly developed durable capacity to form NETs (Figure 1, A and B). We serially assessed NET formation over the first 60 days of extrauterine life for 7 premature neonates. Stimulated NET formation was demonstrable by day 3 ex utero for even the most prematurely born infants (Sup- 
A

\begin{tabular}{|c|c|}
\hline nNIF & $\mathrm{NH}_{2}$-.--------KFNKPFVFLMIEQNTKSPLFMGKVVNPTQ_-COOH \\
\hline CRISPP & 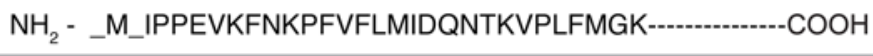 \\
\hline C-terminus A1AT & $\mathrm{NH}_{2}$ - PMSIPPEVKFNKPFVFLMIEQNTKSPLFMGKVVNPTQK-COOH \\
\hline
\end{tabular}

B
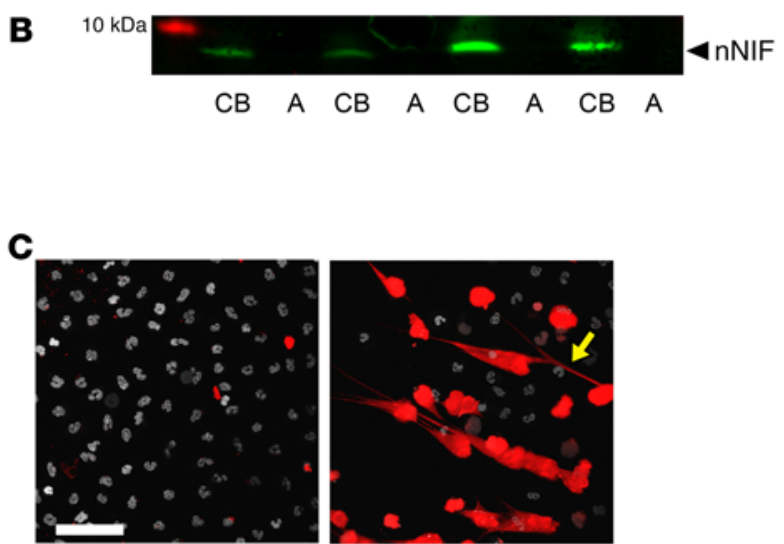

Control

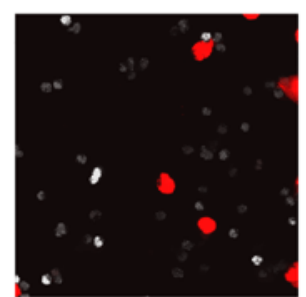

Cord blood plasma/LPS

E

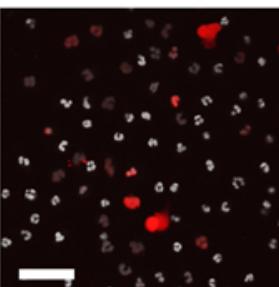

Control

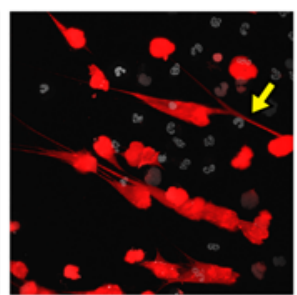

Buffer/LPS

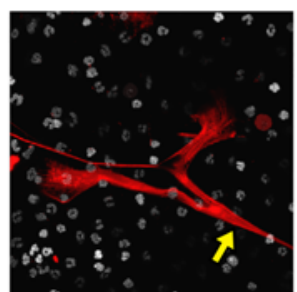

nNIF-depleted

cord blood plasma/LPS

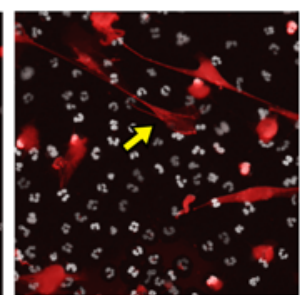

LPS

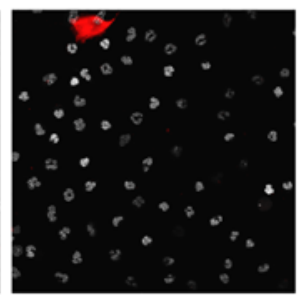

Eluted nNIF/LPS

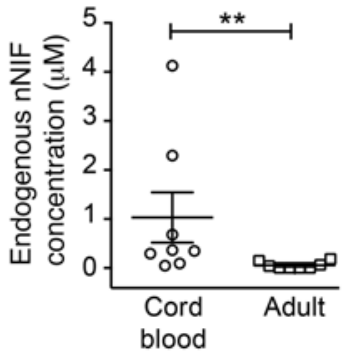

D

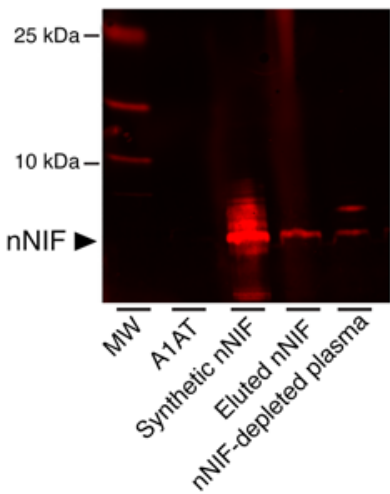

Figure 2. nNIF and related NRPs represent a family of NET-inhibitory peptides. (A) Provisional partial sequence of nNIF from mass spectroscopy and published sequences of CRISPP (35) and A1AT. (B, left panel) Samples of healthy term neonate cord blood plasma $(n=4)$ and adult venous plasma $(n=4)$ were analyzed by Western blotting using a polyclonal antibody against the carboxy terminus of A1AT (full gel shown in Supplemental Figure 1). (B, right panel) Size exclusion of full-length A1AT, quantitative Western blotting with the same polyclonal antibody against the A1AT carboxy terminus, and a standard curve generated using synthetic nNIF (Supplemental Table 2) were used to measure nNIF concentrations in cord blood plasma from preterm neonates and venous plasma from healthy adults ( $n=8$ in each group). Student's $t$ test, ${ }^{* *} P<0.01$. CB, cord blood plasma; A, adult peripheral blood plasma. (C) NET formation by LPS-stimulated (100 ng/ml, 1 hour) adult neutrophils was assessed as in Figure $1 \mathrm{~A}$ after preincubation of the PMNs in control medium, cord blood plasma, cord blood plasma depleted of nNIF using a polyclonal carboxy terminus A1AT antibody coupled to affinity beads or eluate from the affinity beads. This result was consistent in 3 experiments with neutrophils from different donors. Original magnification, $\times 60$. Scale bar: $50 \mu \mathrm{m}$. (D) Full-length A1AT, synthetic nNIF, and samples of depleted cord blood plasma and eluate studied in C were subjected to Western blotting using the carboxy terminus A1AT antibody. Full-length A1AT ( $52 \mathrm{kDa}$ ) was not detected on this $16.5 \%$ Tris-tricine gel due to its size. (E) NET formation (red fluorescence, yellow arrows; scale bar: $50 \mu \mathrm{m}$ ) by LPS-activated adult PMNs was assessed as in Figure $1 \mathrm{~A}$ after preincubation for 1 hour in control medium (second panel), or with full-length A1AT $(2 \mu \mathrm{M})$ or synthetic nNIF $(1 \mathrm{nM})(n=3)$. One-way ANOVA with Tukey's post hoc test. ${ }^{*} P<0.05$, nNIF versus both control medium/LPS and A1AT/LPS.

plemental Table 1; supplemental material available online with this article; doi:10.1172/JCI83873DS1), and maximal NET-forming capacity was achieved between day 3 and day 14 (Figure 1A). Impaired perinatal NET formation is a feature of the neonate:
PMNs isolated from healthy pregnant women immediately before delivery robustly formed NETs (Figure 1C).

Rapid development of NET competency (Figure 1A) suggested that a factor in umbilical cord blood modulates NET formation. To 
A

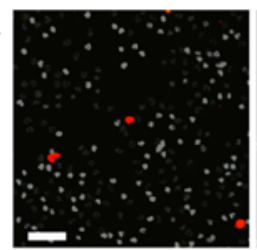

Control

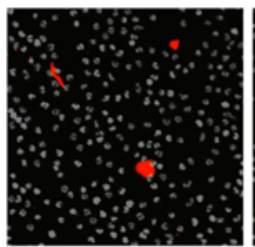

CRISPPI

LPS

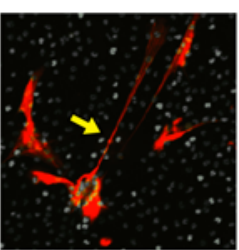

LPS

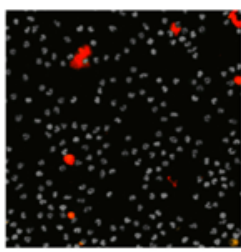

nNIF/

LPS

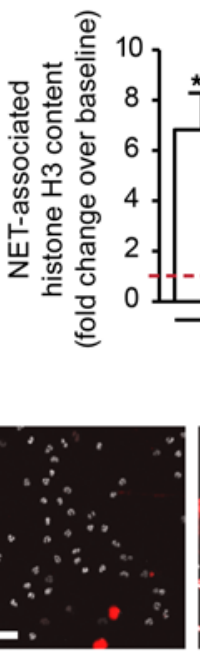

Control

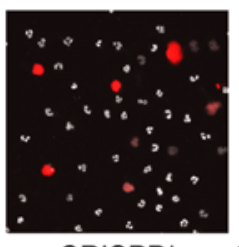

CRISPP/

SA

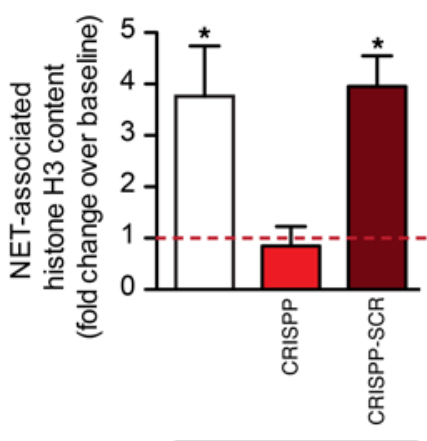

SA
B

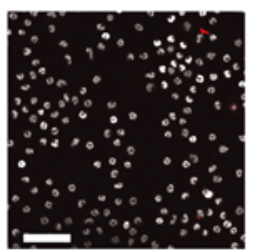

Control

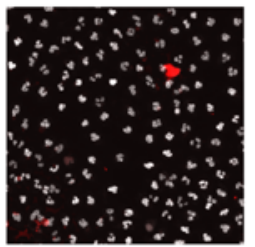

CRISPPI

PMA

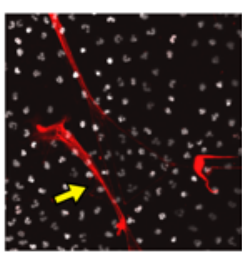

PMA

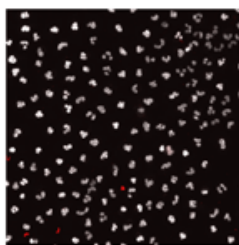

nNIF/

PMA

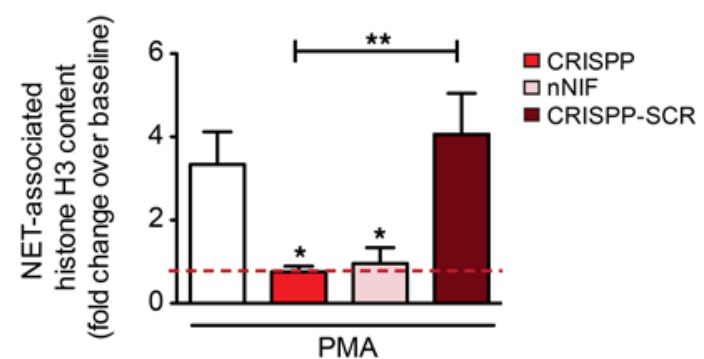

E

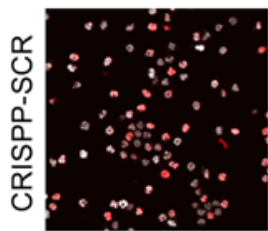

Dengue

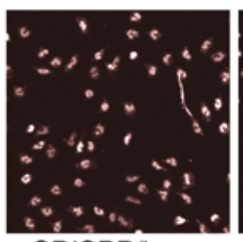

CRISPP/heme

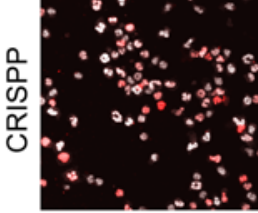

Mock
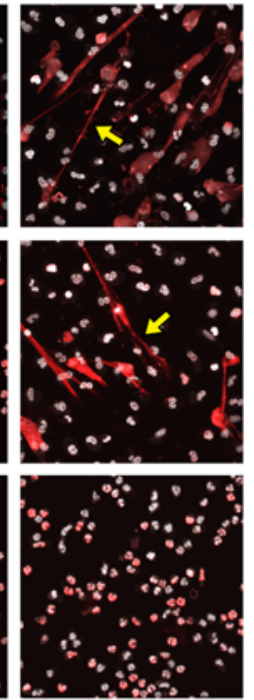

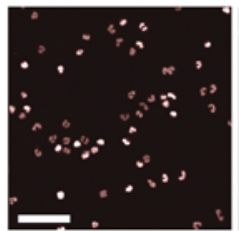

Control

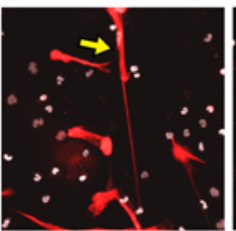

LPS

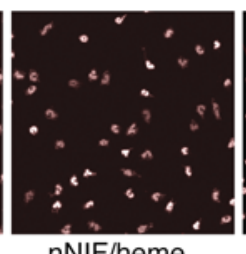

nNIF/heme heme

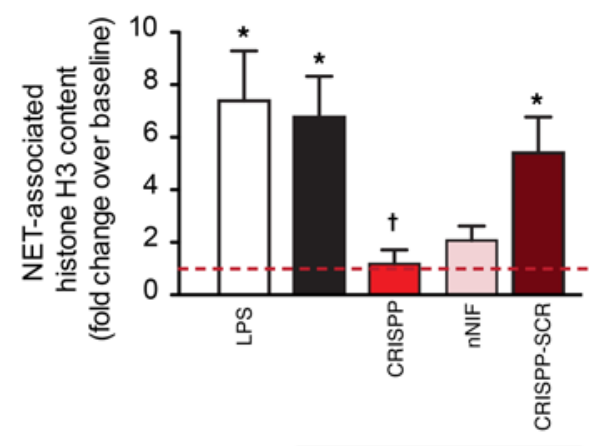

Heme 
Figure 3. nNIF and the NRP CRISPP inhibit in vitro NET formation triggered by a spectrum of NET-inducing agonists. Neutrophils from venous blood of healthy adults were preincubated in medium alone or with nNIF, CRISPP, or CRISPP-SCR (all $1 \mathrm{nM}$ ) for 1 hour, then activated with the indicated agonists ( $n \geq 3$ for each). NET formation (red fluorescence, yellow arrows) was assessed after 1 hour of incubation as in Figure $1 \mathrm{~A}$. All data are represented as \pm SEM. In A, B, C, and E, control values arbitrarily set at 1 are indicated by dashed red lines. One-way ANOVA with Tukey's post hoc testing was applied in A, B, C, and E. nNIF was not studied in C or D. (A) LPS, $100 \mathrm{ng} / \mathrm{ml}$. ${ }^{*} P<0.05$, LPS and CRISPP-SCR/LPS compared with control, ${ }^{t} P<0.05$, CRISPP/LPS and nNIF/LPS compared with both LPS and CRISPP-SCR/LPS. (B) PMA (20 nM). ${ }^{*} P<0.05$, both nNIF/PMA and CRISPP/PMA compared with PMA or CRISPP-SCR/PMA; **P $<0.01$, CRISPP/PMA versus CRISPP-SCR/PMA. (C) S. aureus (SA; MOI 100:1). ${ }^{*} P<0.05$, CRISPP/SA compared with SA or CRISPP-SCR/SA. (D) Dengue virus (MOI 0.05:1). Viral culture medium alone served as a "mock" control (left panels) for dengue virus. Following incubation, the PMNs were immediately fixed in the incubation medium ( $2 \%$ PFA) prior to imaging, and quantitation of histone $\mathrm{H} 3$ release was not possible. (E) Heme $(1 \mu \mathrm{M})$. ${ }^{*} P<0.05$, heme, LPS, and CRISPP-SCR/heme versus control; ${ }^{\dagger} P<0.05$, CRISPP/heme versus heme. Original magnification, $\times 20$. Scale bars: $50 \mu \mathrm{m}$.

test this possibility, we performed "switch" experiments in which PMNs from 60-day-old preterm neonates were preincubated with stored, day 0 autologous cord blood plasma or with freshly collected autologous day- 60 venous blood plasma. PMNs from healthy adults were preincubated in day- 0 cord blood plasma or, in parallel, in autologous adult plasma. Preincubation in day- 0 cord blood plasma depressed NET formation by day-60 neonatal PMNs and control adult PMNs stimulated with LPS, whereas freshly isolated autologous plasma did not (Figure 1D). This result, and the time course of NET competency (Figure 1A), is consistent with a cord blood plasma factor that inhibits NET formation and that rapidly decreases in the circulation of the infant after delivery.

Preliminary experiments involving heat denaturation, proteinase $\mathrm{K}$ treatment, and lipid extraction of cord blood plasma to identify the NET inhibitory factor indicated that it is a protein (our unpublished data). We, therefore, examined the proteomes of day- 0 cord blood plasma and day- 28 venous blood plasma from a preterm infant whose NET-forming capacity was determined in experiments summarized in Figure 1A. 2D gel electrophoresis demonstrated protein and peptide clusters with differential representations in cord blood and day-28 plasma samples. Trypsin digest and tandem mass spectroscopic analysis of proteins from one of the clusters, using the NCBI human trypsin-specific database (ftp://ftp.ncbi.nlm.nih.gov/blast/db/FASTA/nr.gz), yielded partial or complete sequences including a peptide in cord blood plasma with a predicted molecular mass of approximately 4 $\mathrm{kDa}$. Its sequence was identical to the sequence in the carboxy terminus of $\alpha$-1-antitrypsin (A1AT) (Figure 2A), a known 52-kDa plasma protease inhibitor with antiinflammatory and immunomodulatory properties $(31,32)$. Using Western blotting with a polyclonal antibody raised against the carboxy terminal 18 amino acids of A1AT, we found an approximately 4- to 6-kDa peptide in term infant cord blood plasma in much greater abundance than in venous plasma from healthy adults (Figure $2 \mathrm{~B}$ ) that we provisionally termed neonatal NET-inhibitory factor (nNIF). We then immunodepleted cord blood plasma using the anti-A1AT carboxy terminus antibody immobilized on affinity resin beads. Depleted plasma and peptides eluted from the affinity beads were examined for NET-inhibitory activity. Unaltered cord blood plasma inhibited NET formation by LPS-stimulated adult PMNs, as in previous experiments (Figure 1C), as did peptides eluted from the immunoaffinity beads, whereas immunodepleted plasma did not (Figure 2C). A 4- to 6-kDa candidate nNIF peptide was found in much higher quantity in the affinity purification eluate compared with the depleted plasma (Figure 2D). In parallel, we synthesized the 29-amino acid peptide detected in cord blood plasma (Figure 2A) and found that this synthetic nNIF had potent NET-inhibitory activity (Figure 2E). A scrambled control peptide (nNIF-SCR; Supplemental Table 2) did not. These results demonstrate that nNIF, or a larger protein that encompasses it, is an endogenous inhibitor of NET formation in cord blood plasma from preterm and term neonates (Figure 2B). Commercially available, active, full-length A1AT purified from human plasma and recombinant A1AT did not inhibit NET formation (Figure 2E), consistent with previous reports $(33,34)$, indicating that intact A1AT does not contribute to NET-inhibitory activity.

With an assay utilizing quantitative Western blotting with the anti-A1AT carboxy terminus antibody and a standard curve constructed with different concentrations of synthetic nNIF, we detected nNIF in preterm cord blood plasma samples, whereas it was undetectable, or detectable in only trace levels, in plasma from healthy adults (Figure 2B). Using the same assay, we did not detect a peptide of appropriate molecular mass in plasma samples from adult subjects $(n=10)$ with chronic inflammatory syndromes (granulomatosis with polyangiitis, giant cell arteritis, or rheumatoid arthritis) that might conceivably induce NET regulatory factors (our unpublished data). Thus, nNIF may be restricted to placental blood and blood of neonates in the first few days of life.

$n$ NIF and nNIF-related peptides are a family of previously unrecognized PMN modulators. We identified additional NET inhibitory peptides based on sequence analysis of nNIF. nNIF has substantial similarity to cancer-associated SCM recognition, immune defense suppression, and serine protease protection peptide (CRISPP) (Figure $2 \mathrm{~A}$ ), a consensus peptide based on factors present in the blood of patients with cancer (35-37). We synthesized CRISPP and a scrambled control peptide (CRISPP-SCR) and found that CRISPP inhibits NET formation triggered by LPS, as does nNIF, whereas CRISPP-SCR does not (Figure 3A). nNIF and CRISPP inhibited NET formation by neutrophils isolated by Ficoll-Paque and differential centrifugation in addition to PMNs isolated by positive immunoselection. Posttreatment protocols demonstrated that nNIF and CRISPP do not degrade or dismantle previously formed NETs (Figure 4). Thus, they differ from DNases, which disrupt NETs after they are formed $(2,3,9,15,38)$. We also examined a previously described 44-amino acid carboxy terminus cleavage fragment of A1AT, A1ATm ${ }^{358}$, which is bound to matrix in the human placenta and overlaps in sequence with nNIF $(39,40)$ (Supplemental Table 2). We synthesized A1ATm ${ }^{358}$ and found that it inhibits NET formation, although with lesser potency than nNIF (Figure 5).

Together, these observations indicate that nNIF, CRISPP, and $\mathrm{A}^{\mathrm{ATTm}}{ }^{358}$ represent a previously unrecognized family of nNIFrelated peptides (NRPs) that modulate NET formation (Figures $1-5)$. The presence of NRPs in umbilical blood (nNIF), placenta $\left(\mathrm{A} 1 \mathrm{ATm}{ }^{358}\right)$, and, in some cases, adult plasma (CRISPP-related 
A
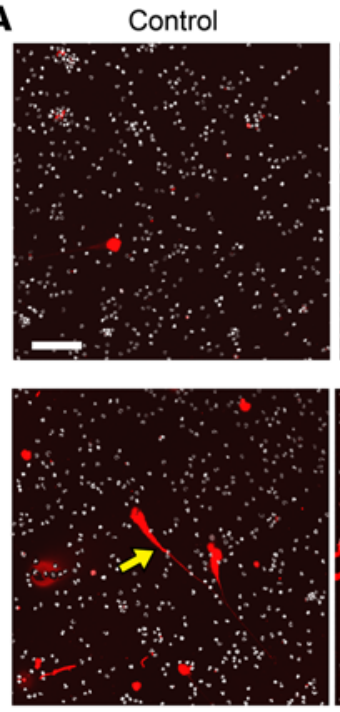

0 min
LPS
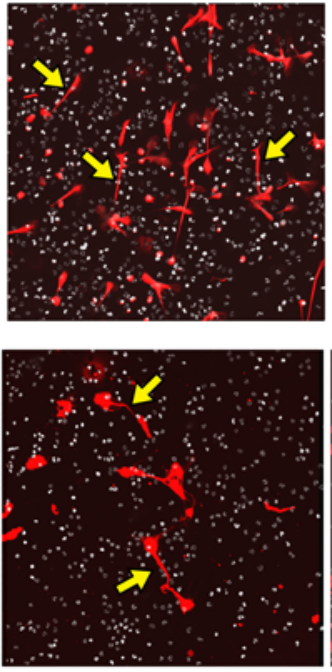

$30 \mathrm{~min}$

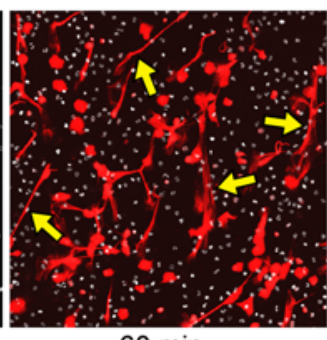

$60 \mathrm{~min}$
Figure 4. NRPs do not dismantle NETs. (A) Neutrophils isolated from venous blood of healthy adults were incubated in medium alone (control) or activated with LPS (100 ng/ml). CRISPP ( $1 \mathrm{nM}$ ) was added at 0,30 , or 60 minutes after LPS, and the presence of NETs (red fluorescence, yellow arrows) was assessed by live cell imaging as in Figure $1 \mathrm{~A}$ after an additional 1 hour of incubation. The images are representative of 3 separate experiments. Original magnification, $\times 20$. Scale bars: 100 $\mu \mathrm{m}$. (B) Isolated adult neutrophils were stimulated with LPS (100 ng/ml, 1 hour). DNase (3.78 U/ml), nNIF (1 nM), or CRISPP ( $1 \mathrm{nM})$ was then added, and NETs were imaged as in $\mathbf{A}$ after an additional 1 hour of incubation. In a second experiment, NETs were also intact after treatment with nNIF or CRISPP, but dismantled by DNase. Original magnification, $\times 60$. Scale bars: $20 \mu \mathrm{m}$.

Time to CRISPP addition after LPS stimulation

B

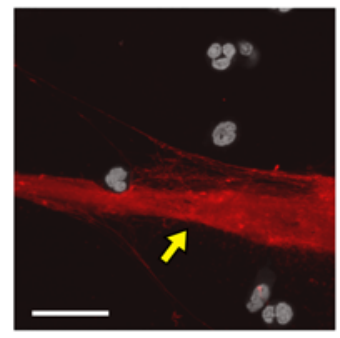

DNase

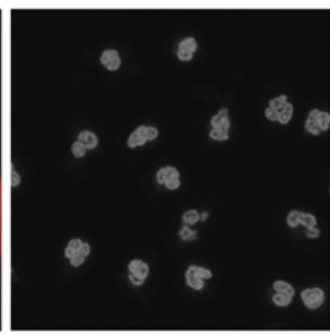

nNIF

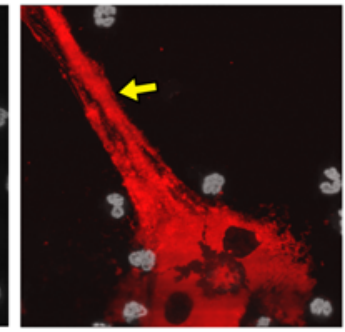

CRISPP

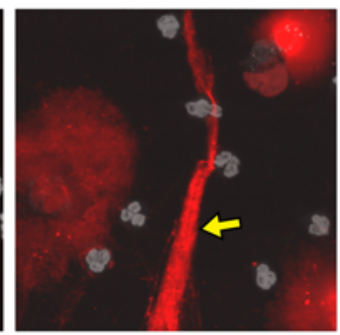

peptides) suggests that NET inhibitory factors may be broadly distributed and that other NRPs may be identified.

CRISPP and NNIF inhibit NET formation induced by a spectrum of NET-triggering agonists. We examined the inhibitory activity of NRPs when NET formation is induced by diverse agonists, focusing on nNIF and CRISPP. Both inhibited LPS-stimulated NET deployment in multiple experiments (Figure 2E, Figure 3A, and Supplemental Figure 2). PMA is commonly employed as a potent nonphysiologic agonist to induce NET formation in vitro $(1,6,33$, 41). nNIF and CRISPP, but not CRISPP-SCR, blocked PMA-stimulated NET deployment (Figure 3B). CRISPP also inhibited NET formation induced by live Staphylococcus aureus $(2,9,28,41)$ (Figure $3 \mathrm{C}$ ). This result suggests that NRPs inhibit "vital" NETosis in addition to "suicidal" NETosis, as is triggered by PMA $(3,41)$, since $S$. aureus is reported to release NETs by chromatin decondensation and vesicular export without neutrophil lysis $(3,5,42)$. We also examined the ability of NRPs to inhibit NETosis induced by other pathogens and found that CRISPP inhibited NET generation stimulated by dengue virus (Figure 3D). Several viruses trigger NET deployment (43-45), but dengue, which interacts with ligands on myeloid cells (46), has not previously been reported as having this activity. To further explore the inhibitory activities of nNIF and NRPs, we examined heme, an endogenous damageassociated molecular pattern (DAMP) and toxin (47) that induced NETs in a murine model of sickle cell vasculopathy (17). We found that heme triggers NET formation by human PMNs and that nNIF and CRISPP inhibit this response (Figure 3E). Thus, NRPs inhibit NET deployment induced by microbes and microbial toxins, host-derived DAMPs, and pharmacologic agonists.

NRPs selectively inhibit NET formation without interrupting other key neutrophil antimicrobial functions or platelet responses. We examined total, phagocytic, and NET-mediated PMN killing of a pathogenic strain of Escherichia coli using bacterial killing assays and found that CRISPP depressed extracellular NET-mediated and total bacterial killing, but that phagocytic intracellular killing was not altered (Figure 6A). In additional incubations employing nNIF or CRISPP, the NRPs did not inhibit generation of ROS, phagocytosis, or IL-8-induced chemotaxis in Boyden chambers (Figure 6, B-D). Although each peptide was not tested in all assays, these experiments indicate that the NRPs selectively inhibit NET formation while leaving other key antimicrobial activities of PMNs intact. In addition, we found, using flow cytometry, that CRISPP does not inhibit surface translocation of P-selectin by thrombinstimulated platelets or formation of heterotypic aggregates by activated human platelets and PMNs (Figure 6, E and F). These and other functions of activated platelets are important in antimicrobial defense (48). In addition, interaction of activated platelets with neutrophils induces NET formation $(13,15)$. Figure $6, \mathrm{E}$ and F, indicates that PMNs, but not platelets, are cellular targets for NRPs and that platelet inflammatory activities are not disrupted by NRPs.

$n N I F$ and CRISPP inhibit nuclear chromatin decondensation and histone citrullination in activated neutrophils. We used nNIF 
A
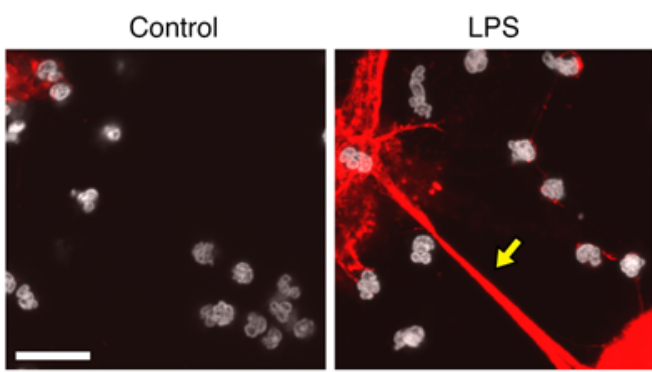

B
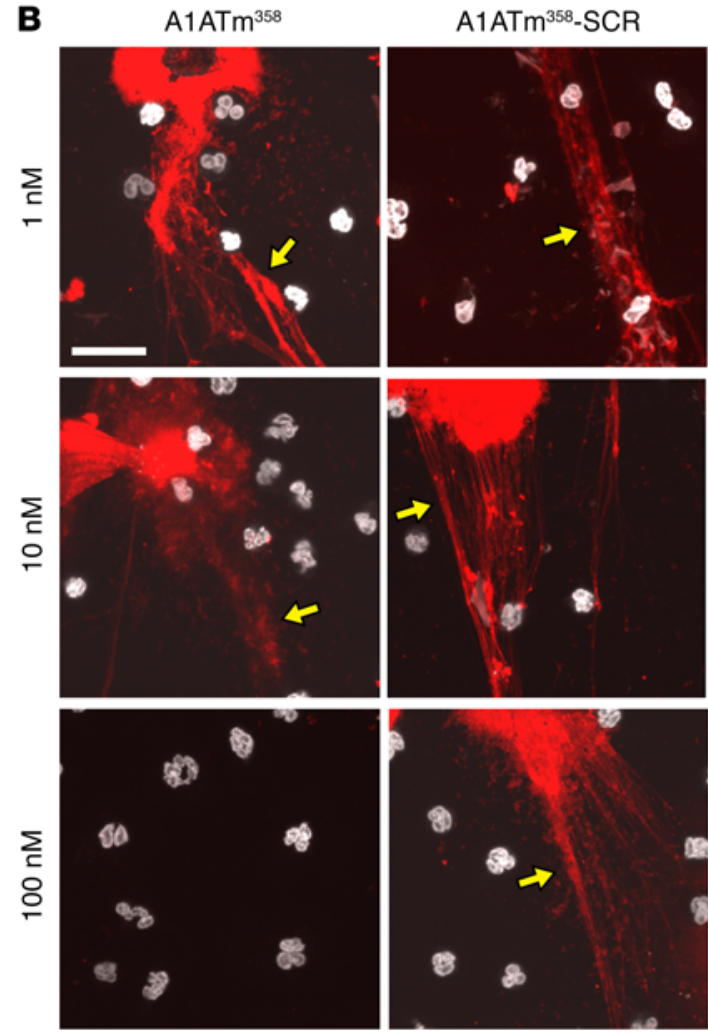

and CRISPP as probes to dissect mechanisms of action for NRPs. Generation of ROS is thought to be essential in many, but not all, pathways that mediate NET formation $(2,3,6,18,28,33,49)$, but was not inhibited by CRISPP (Figure 6B). Consistent with this result, our previous observations indicated that ROS supplementation is not sufficient to restore NET competency to neonatal PMNs (28), suggesting that nNIF also acts at a regulatory step or steps different from those influenced by ROS.

Decondensation of nuclear chromatin is a pivotal event that is required for NET formation (1-3, 5, 6, 33, 41, 49). We found that PMA induces decondensation and loss of lobulation of PMN nuclei (Figure 7A), as previously reported (6). The number of decondensed nuclei was dramatically reduced by nNIF and CRISPP, but not by nNIF-SCR or CRISPP-SCR (Figure 7A). Neutrophil chromatin decondensation is mediated by PAD4, which weakens histone-DNA binding by catalyzing conversion of histone arginines to citrullines $(1,7,8)$. Consistent with this, an irreversible inhibitor of PAD4, Cl-amidine (8), blocked nuclear decondensation under the conditions of our experiments (Figure 7A). We then employed a cell-free PAD4 assay and found that nNIF blocked its activity, as

Figure 5. A1ATm ${ }^{358}$ inhibits NET formation. (A) Isolated adult neutrophils were preincubated in medium (1 hour) and then incubated alone (control) or with LPS $(100 \mathrm{ng} / \mathrm{ml}, 1$ hour), followed by live cell imaging as in Figure $1 \mathrm{~A}$. Original magnification, $\times 60$. Scale bars: $20 \mu \mathrm{m}$. Red fluorescence, yellow arrows $=$ NETs. $($ B) In parallel, neutrophils were preincubated with synthesized $A 1 A T^{358}$ or scrambled $A 1 A T^{358}$ (A1ATm $\left.{ }^{358}-S C R\right)(1,10$, or $100 \mathrm{nM}, 1$ hour) and activated with LPS; NETs were assessed by live cell imaging after incubation for 1 hour. A second experiment yielded a similar concentration-dependent pattern of inhibition by $A 1 A \mathrm{Tm}^{358}$ but not A1ATm ${ }^{358}$-SCR. Original magnification, $\times 60$. Scale bars: $20 \mu \mathrm{m}$.

did $\mathrm{Cl}$-amidine used as a control (Figure 7C). In an initial comparison of NRPs, the order of potency of inhibition of PAD4 was nNIF $\approx \mathrm{CRISPP}>\mathrm{A}_{\mathrm{ATm}}{ }^{358}$, which is the same as their relative inhibition of NETosis. In parallel, we examined nuclear histone $\mathrm{H} 3$ citrullination in activated neutrophils $(1,8)$ and found rapid citrullination detected within 15 minutes of activation with PMA. This was inhibited by nNIF and by $\mathrm{Cl}$-amidine (Figure 7D), suggesting that NRPs act at this step to block nuclear decondensation (Figure 7A).

Neutrophil elastase (NE) is also implicated in nuclear decondensation and NET formation (1, 2, 6, 9, 49). An NE inhibitor, sivelestat, blocks nuclear decondensation in vitro (Supplemental Figure 4). We synthesized CRISPP and CRISPP-SCR with FLAG tags added to the carboxy terminus of each peptide (CRISPP-F, CRISPP-SCR-F) and found that both are internalized by activated neutrophils (Figure 7E), that CRISPP-F inhibits NET formation (Supplemental Figure 3), and in a protein proximity assay, that CRISPP-F is initially localized within $40 \mathrm{~nm}$ of NE (our unpublished experiments). This suggested that NRPs may block actions of NE in NETosis. In in vitro assays, however, neither nNIF nor CRISPP inhibited NE activity (Supplemental Figure 4 and our unpublished experiments).

NRPs inhibit NET formation in vivo and alter outcomes in systemic inflammation. To determine whether NNIF and NRPs inhibit NET formation in vivo, we established a new model of in vivo NETosis using i.p. infection of C57BL/6 mice with a clinical isolate of E. coli. Three hours after inoculation, live cell imaging of peritoneal fluid samples demonstrated robust NET formation. In addition, we observed deposition of NETs on the serosal surface of the peritoneal membranes. nNIF and CRISPP, but not CRISPP-SCR, inhibited NET formation by peritoneal fluid PMNs (Figure 8A) and deployment of NETs on the peritoneal surface (Figure 8B). Active peritonitis was demonstrated with increased neutrophil numbers and bacterial counts (Figure 8, C and D). The number of PMNs was greater in samples from CRISPP-treated animals, and the trend was to greater numbers in nNIF-treated mice (Figure 8C), potentially due to inhibition of lytic NETosis $(2,3)$. The number of E. coli CFUs was also greater in samples from NRP-treated animals than in those treated with the CRISPP-SCR control (Figure 8D), suggesting decreased NET-mediated bacterial killing. nNIF and CRISPP also inhibited peritoneal NET formation in Swiss Webster mice infected with E.coli (Figure 8, E and F), suggesting that this result is generalizable across mouse backgrounds.

In a second model, we found that i.p. LPS triggers peritoneal NET formation in C57BL/6 mice, although not as robustly as do live E. coli, and that LPS-induced peritoneal NET formation was inhibited by nNIF and CRISPP, but not CRISPP-SCR (our unpublished 
A

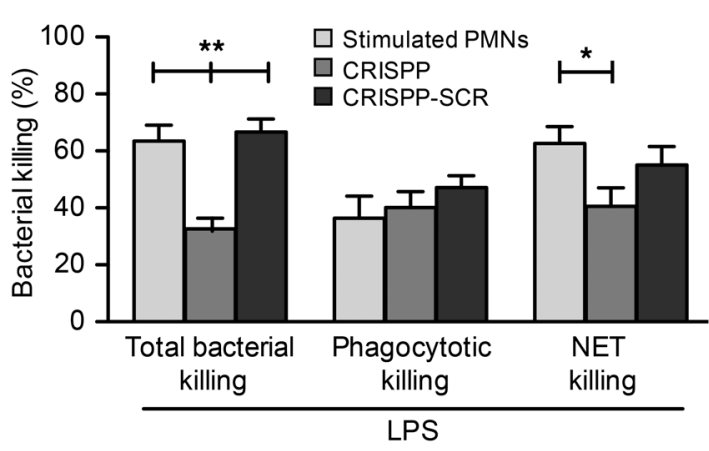

C

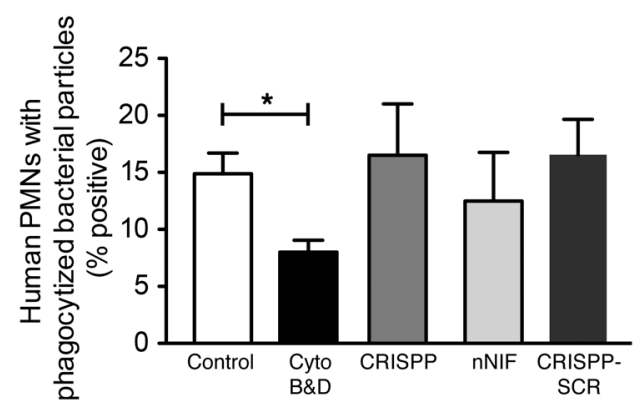

B

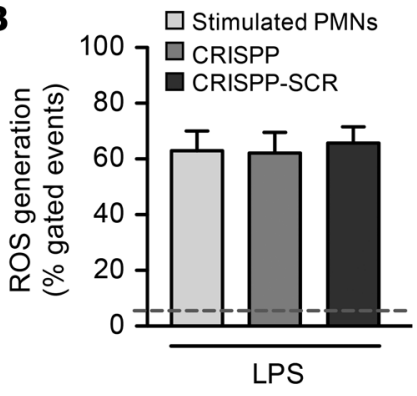

E

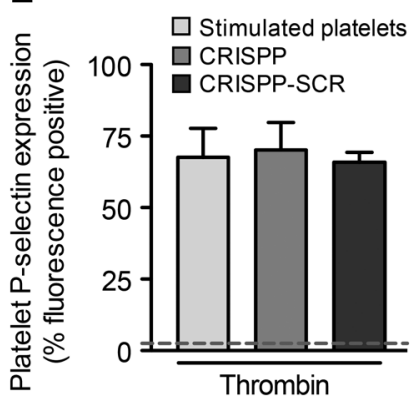

$\mathbf{F}$

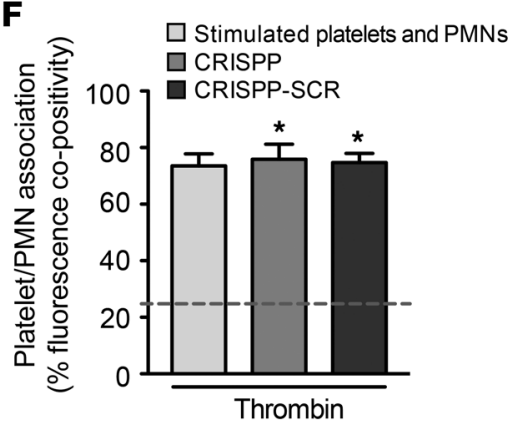

Figure 6. NRPs selectively inhibit NET formation. Isolated adult neutrophils or platelets were preincubated with buffer or with CRISPP, nNIF, or CRISPP-SCR (1 nM, 1 hour for each), followed by measurement of functional responses. A minimum of 3 separate assays were completed for each response. Error bars represent SEM. Tukey's post hoc testing was applied in B, D, E, and F. (A) After preincubation, neutrophils were stimulated with LPS $(100 \mathrm{ng} / \mathrm{ml})$ to trigger NET formation and incubated with a pathogenic isolate of E. coli. Total, phagocytic, and NET-mediated bacterial killing were measured (28). One-way ANOVA with Bonferroni's post hoc testing. ${ }^{*} P<0.05 ;{ }^{*} P<0.01$. (B) Reactive oxygen species generation was measured by dihydrorhodamine detection after LPS stimulation (100 ng/ml, 1 hour). (C) Phagocytosis of fluorescently labeled $E$. coli bioparticles was measured by microscopy after a 4 hour incubation. Treatment with cytochalasin $B$ and $D$ served as a control for inhibition of phagocytosis. Student's $t$ test. ${ }^{*} P<0.05$. (D) Chemotaxis in response to $\mathrm{IL}-8(2 \mathrm{ng} / \mathrm{ml})$ was examined in a Boyden chamber assay. The dashed line indicates the response to IL-8 alone arbitrarily set at 1. (E) Surface translocation of $P$ selectin on platelets activated by thrombin $(0.1 \mathrm{U} / \mathrm{ml})$ was measured by flow cytometry. The dashed line indicates surface $P$ selectin on unstimulated platelets. (F) Formation of platelet-neutrophil aggregates was measured after mixing of platelets activated with thrombin $(0.1 \mathrm{U} / \mathrm{ml})$ and neutrophils activated with LPS (100 ng/ml). The dashed line indicates control aggregate formation in response to LPS stimulation of the PMNs alone. ${ }^{*} P<0.05$, CRISPP and CRISPP-SCR compared with control.

data). Intravascular NETs have been observed in mice challenged with i.p. LPS $(14,50)$; these cause tissue damage and contribute to mortality when they are induced by i.v. LPS, bacteria, or other agonists $(9,12,14,15,17)$. Therefore, we examined mortality in mice given i.p. LPS and found that all animals treated with CRISPP $(n=6)$, but only $30 \%$ of those treated with CRISPP-SCR $(n=6)$, were alive when the experiment was terminated at 50 hours $(P<0.02)$. In a second experiment, in which nNIF was also examined and that was extended to 72 hours, there was reduced mortality in the NRP-treated groups challenged with LPS compared with mice treated with CRISPP-SCR or LPS alone ( $n=10$ for each group) at 50 hours. Figure 9A illustrates combined data from the 2 experiments. At 72 hours in the second experiment, the survival advantage provided by $\mathrm{nNIF}$ was durable ( $P=0.007$ compared with LPS alone), whereas that of CRISPP was not. This may be due to differences in pharmacokinetics or half-lives of nNIF and CRISPP under these conditions.

We also performed similar experiments using the cecal ligation and puncture (CLP) model of polymicrobial sepsis (51-53). Mice treated with nNIF had lower clinical illness scores (52) at 24 hours and significantly increased survival at 144 hours after CLP compared with nNIF-SCRtreated animals (Figure 9, B and C). Together, these experiments (Figure 8 and Figure 9) demonstrate that nNIF and CRISPP inhibit NET formation in vivo and provide initial evidence that they have beneficial effects in models of systemic sterile inflammation and infection in which NET formation may influence tissue injury and mortality $(9,13,14,53)$.

\section{Discussion}

Inflammatory pathways and immune mechanisms have checkpoints and modulatory brakes that prevent inappropriate initiation or unregulated propagation of effector events, which could otherwise cause pathologic collateral injury to the host $(54,55)$. Tight control and modulation of inflammatory responses appear to be particularly important in the fetus and neonate, but the cellular and molecular mechanisms involved remain incompletely defined $(25-27,56,57)$. nNIF in umbilical cord blood and $\mathrm{A}^{\mathrm{ATT}} \mathrm{m}^{358}$ in the placental matrix may represent regulatory factors that modulate NETosis in the perinatal milieu. Placental IL-8, a NETosis-inducing chemokine (41), and syncytiotrophoblast microparticles trigger NET formation in vitro, and NETs are present in placentas of women with preeclampsia (58). Thus NET-inducing stimuli appear to be generated at the maternal-fetal 
interface, suggesting that unregulated NET formation can cause inflammatory pathology in the fetomaternal environment. Excessive intrapartum NET formation could also have additional negative consequences, including long-term neonatal immune dysregulation $(2,3,25,26,56,59)$. Immediately after delivery, the neonate is at risk for NET-mediated vascular injury and thrombosis $(1-3,9$, $13,19,38)$ triggered by microbial colonization $(22,23)$ and consequent neutrophil mobilization (24) if NET formation is not tightly controlled. nNIF in neonatal plasma and the related NRP A1ATm ${ }^{358}$ in the placental interstitium (40) represent potential "stop signals" (54) that selectively limit NET formation before and immediately after birth. Rapid development of full NET competency by neonatal PMNs (Figure 1) and decreased nNIF in neonatal blood in the first few days of extrauterine life parallel establishment of the resident microbiota of the human infant $(22,23)$ and suggest that these are regulated features of immune development. In our initial screens, nNIF was not detected or was minimally present in plasma samples from healthy adults or adult patients with chronic inflammatory syndromes (Figure 2B; our unpublished data). This suggests that nNIF expression may largely be a feature of the fetus and neonate, as are certain other immunoregulatory mechanisms (25-27). Additional studies of healthy and diseased adults, potentially with more sensitive assay methods, will be required to resolve this issue.

Our studies and previous observations suggest that nNIF and $\mathrm{A}^{\mathrm{ATTm}}{ }^{358}$ are generated by proteolytic cleavage of A1AT in the placenta. A1AT is abundant in human placental tissue compartments $(60,61)$. It has been proposed that progressive proteolytic cleavage of A1AT occurs in the placenta, and proteases that mediate enzymatic fragmentation of A1AT to A1ATm ${ }^{358}$ in vitro are known $(39,62,63)$, although a specific placental protease has not been identified. A protease that is increased in human placental syncytiotrophoblasts in the third trimester of pregnancy, high temperature requirement protease 1, cleaves A1AT in the carboxy terminus (61). Together, these findings suggest a mechanism for generation of biologically active fragments of A1AT in the placenta that would no longer be active after delivery and separation of the neonate. Nevertheless, the mechanism of generation of nNIF in the fetomaternal environment and the factors that control levels of nNIF in cord and postnatal blood (Figure 2) remain to be defined.

In addition to nNIF and A1ATm ${ }^{358}$, we identified CRISPP as an NRP. CRISPP-related peptides have been detected in plasma from patients with multiple types of cancer (35-37), but have not been linked to regulation of NETosis. NET formation facilitates experimental metastasis (64) and may also contribute to outcomes in cancer-associated infection and sepsis. Thus, endogenous CRISPP-related peptides may have significant influences on neoplastic complications by inhibiting formation of NETs, an issue that merits investigation. In this study, however, we focused on characterizing CRISPP as a lead NRP that, together with nNIF, may have broad relevance and utility in exploration of NET biology.

We found that nNIF and CRISPP inhibit in vitro NET deployment induced by $S$. aureus, the bacterial toxin LPS, a previously unrecognized viral trigger, dengue, a host-derived DAMP, heme, and the potent pharmacologic agonist, PMA. While we have not yet examined fungal agonists $(2,11,65)$, this analysis indicates that NRPs interrupt NET formation triggered by diverse stimuli that may be mediated by distinct activation pathways (1). Fur- thermore, nNIF and CRISPP inhibited in vivo NET formation in a murine model of E. coli peritonitis (Figure 8). Here, NET formation is likely induced by the bacteria, LPS, and host-derived mediators, suggesting that NRPs can inhibit NET deployment by neutrophils stimulated by multiple agonists that act in combinational fashion in a complex inflammatory milieu and that, perhaps, induce NET formation via more than one pathway simultaneously (3). Validation of nNIF and CRISPP as inhibitors of NET deployment in this model (Figure 8) also complements analysis of in vitro inhibition (Figure 3), since it is suggested that pathways to NET formation vary in vivo and in vitro (1).

We utilized nNIF and CRISPP as probes to explore the mechanism or mechanisms by which NRPs inhibit NET formation. While NETosis induced by a number of agonists involves ROS generation (1-4, 6, 18, 49, 66), current (Figure 6B) and previous (28) experiments indicate that NRPs act at a different step or steps. Based on all studies to date, chromatin decondensation is critical in NET deployment regardless of the agonist $(1-3,5,6,33$, $42,49)$. We found that nNIF and CRISPP inhibit loss of lobules and expansion of nuclei in PMA-stimulated neutrophils (Figure 7A and Supplemental Figure 4) in an assay based on earlier studies of chromatin decondensation in NETosis (6). Neutrophil heterochromatin decondensation is mediated by PAD4, which catalyzes conversion of histone arginines to citrullines with consequent weakening of histone-DNA binding and unwinding of nucleosomes (1, 7-9). In a cell-free assay of PAD4 activity based on examination of a synthetic substrate, we found inhibition by nNIF in parallel with Cl-amidine, an established PAD4 inhibitor (Figure 7C). This is consistent with inhibition of nuclear decondensation by each agent (Figure 7A). In live, intact human neutrophils activated with PMA, nNIF and Cl-amidine inhibited nuclear histone citrullination, which, as expected, occurred before nuclear decondensation (Figure 7D). In preliminary assays, CRISPP also inhibited PAD4 activity and nuclear histone citrullination (our unpublished experiments). Together, these findings indicate that NRPs inhibit nuclear decondensation and NET formation at least in part by inhibiting PAD4 activity and nuclear histone examination. NE is also thought to have a critical role in NETosis (1). NE mediates nuclear histone cleavage in PMA-activated neutrophils (6), inhibitors of NE activity block nuclear decondensation $(1,6,33)$ (Supplemental Figure 4) and NET formation in vivo (64), endogenous regulators of NE influence NETosis $(33,67)$, and NET generation is impaired in NE-deficient mice (9). Nevertheless, we found that NRPs do not directly inhibit NE activity in in vitro assays (Supplemental Figure 4 and our unpublished experiments). We do not at this time exclude the possibility that NRPs interrupt NE-mediated events in NETosis pathways in other ways, however. A FLAGtagged construct of CRISPP that inhibits NET formation (Supplemental Figure 3) was internalized by activated PMNs (Figure 7E) and was closely localized near NE in the neutrophil cytoplasm (our unpublished experiments), suggesting that NRPs may have more than one site and mechanism of action.

A central question in current inflammatory biology is whether NETs primarily protect the host from pathogens, and, if so, under what infectious circumstances, or whether they, in contrast, are primarily effectors or modulators of inflammatory injury (1-3, 9, $13-20,66,68)$. Selective inhibitors of NET formation may be par- 
A

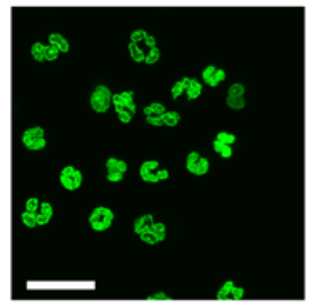

Control

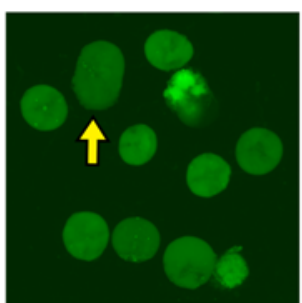

nNIF-SCR PMA

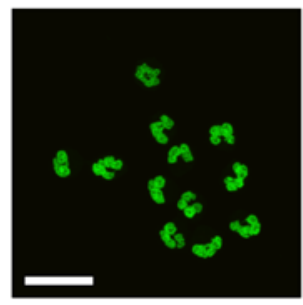

Control

D

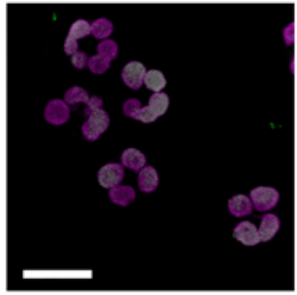

Control

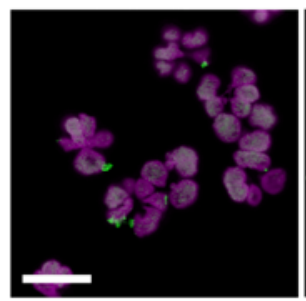

nNIF PMA

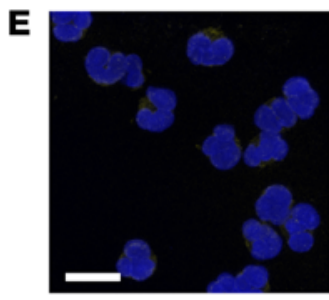

IgG control

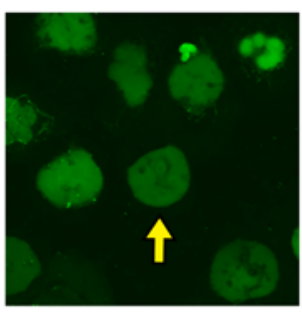

PMA

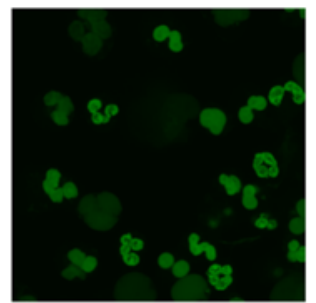

CRISPP PMA

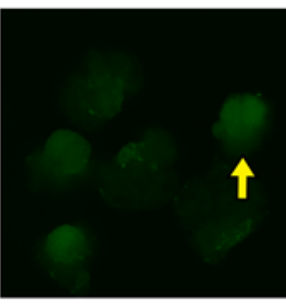

PMA

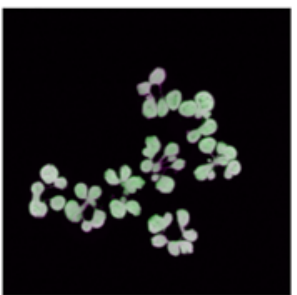

PMA

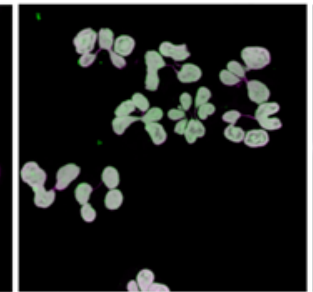

nNIF-SCR PMA

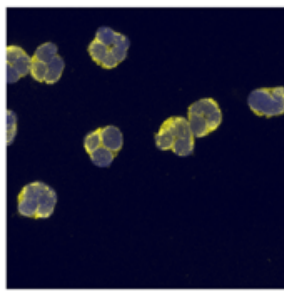

CRISPP-FLAG

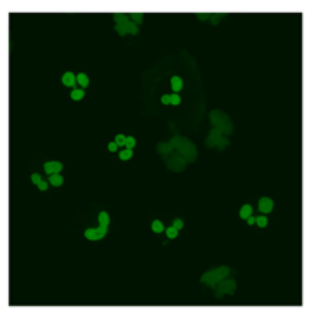

nNIF PMA

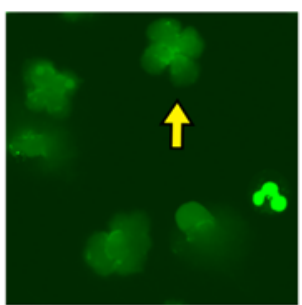

CRISPP-SCR PMA

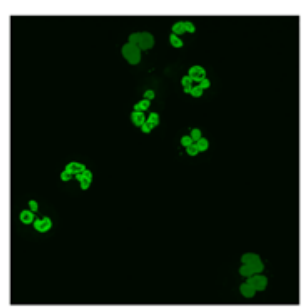

Cl-amidine PMA

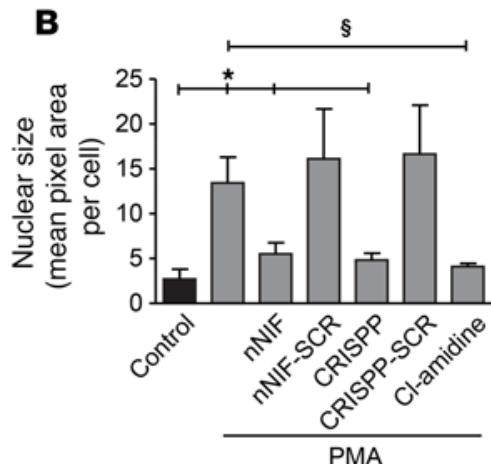

C

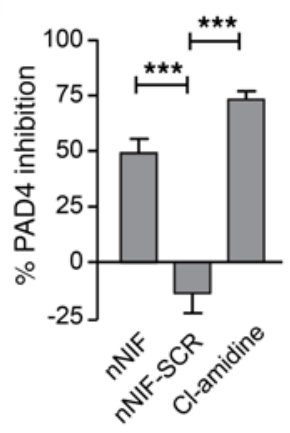

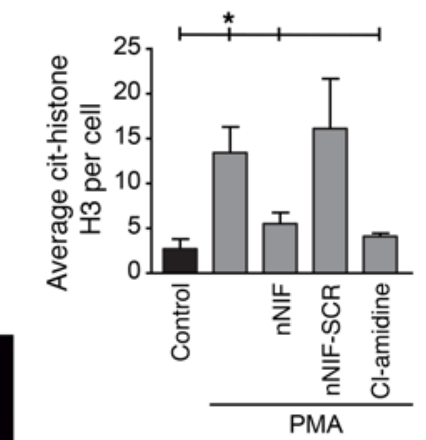

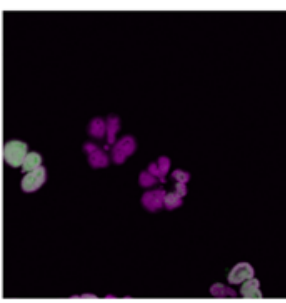

Cl-amidine PMA 
Figure 7. NRPs inhibit nuclear decondensation and histone citrullination in activated neutrophils. (A) Neutrophils were preincubated in medium alone, with nNIF, nNIF-SCR, CRISPP, or CRISPP-SCR (all $1 \mathrm{nM}$ ), or with the irreversible PAD4 inhibitor $\mathrm{Cl}$-amidine $(10 \mu \mathrm{M})$ for 1 hour, treated with PMA ( $20 \mathrm{nM})$, and then incubated on poly-L-lysine-coated coverslips for 2 hours, followed by examination for nuclear decondensation (arrows) by live cell imaging. Green fluorescence, nuclear DNA. Original magnification, $\times 60$. Scale bars: $20 \mu \mathrm{m}$. (B) Nuclear areas were measured $(n=4)$ using ImageJ software (mean nuclear pixel area per cell \pm SEM). Paired Student's $t$ test. ${ }^{*} P<0.05 ;{ }^{\circledR} P=0.057$. (C) nNIF (1 nM), nNIF-SCR (1 nM), or Cl-amidine (10 $\mu \mathrm{M})$ was examined in a cell-free deimination assay employing recombinant PAD4 and a synthetic substrate. One-way ANOVA with Tukey's post hoc testing. ${ }^{* *} P<0.001$. (D) Left panels: neutrophils were preincubated for 30 minutes in medium, with nNIF or nNIF-SCR (both $1 \mathrm{nM}$ ) or with $\mathrm{Cl}$-amidine $(10 \mu \mathrm{M})$, activated for 15 minutes with PMA $(20 \mathrm{nM})$, and citrullinatedhistone $\mathrm{H} 3$ was detected by immunocytochemistry $(n=3)$. Green fluorescence, citrullinated-histone $\mathrm{H3}$; magenta fluorescence, nuclear DNA. Original magnification, $\times 60$. Scale bars: $20 \mu \mathrm{m}$. Right panel: histone $\mathrm{H3}$ citrullination was quantified using Image) software (mean citrullinatedhistone $\mathrm{H} 3$ pixel area per cell $\pm \mathrm{SEM})(n=3)$. One-way ANOVA with Tukey's post hoc testing. ${ }^{*} P<0.05$. (E) Neutrophils were incubated with FLAGtagged CRISPP-FLAG or CRISPP-SCR-FLAC (1 nM for both) for 1 hour, activated with LPS $(100 \mathrm{ng} / \mathrm{ml})$ for a further 2 hours, and then examined by confocal microscopy using an anti-FLAG antibody $(n=3)$. Yellow fluorescence, FLAG tag; blue fluorescence, nuclear counterstain. Original magnification, $\times 60$. Scale bars: $20 \mu \mathrm{m}$. The FLAG-tagged peptides were not internalized by isolated human platelets (our unpublished experiments).

ticularly useful in dissecting this issue in a context-specific fashion and may provide experimental advantages over strategies to dismantle NETs with DNases, which can have incomplete effects under some conditions (9). Notably, nNIF and CRISPP effectively inhibit NET formation by both human and murine neutrophils (Figure 3, Figure 8, and our unpublished experiments), whereas recently reported synthetic inhibitors of PAD4 have differential efficacy as inhibitors of NETosis by human and mouse neutrophils (69). In our initial analysis of outcomes when NRPs are administered in vivo, we examined nNIF and CRISPP in mice challenged with LPS, which causes sterile systemic inflammation, NET formation, organ damage, and mortality $(13,14,50,70)$. The NRPs provided an early survival advantage in this model (Figure 9A), suggesting that NETs are agents of inflammatory damage in the absence of pathogens, and a consequent requirement for their containment and elimination. nNIF also improved mortality in the CLP model of polymicrobial sepsis (Figure 9, B and C), supporting existing evidence that NETs are effectors of collateral vascular and tissue injury in this experimental syndrome (53). The results in both models suggest that NET generation, like other neutrophil effector functions (54), has evolved to contain and eliminate pathogens, but can also injure the host if it is activated by pathologic inflammatory signals in the absence of infection or by microbes in an uncontrolled fashion. Our findings also suggest that NRPs have potential as antiinflammatory therapies (71) in specific syndromes in which NET formation contributes to acute or progressive pathologic inflammation.

\section{Methods}

Animal studies. Swiss Webster and C57BL/6 male mice between the ages of 8 and 12 weeks were purchased from Charles River Laboratories or Jackson Laboratories for all experiments. Mice were housed in specific pathogen-free microisolator cages that were located in a room maintaining a constant temperature and on a 12-hour light/12-hour dark cycle. All treatment groups were weight matched and randomized to treatment at the initiation of an experiment. The researchers conducting the experiments were blinded to the experimental groups during testing. No inclusion or exclusion criteria were used in designing the experiments.

Reagents. Lipopolysaccharide (E. coli serotype 0111:134 and Salmonella enteriditis), poly-L-lysine, cytochalasin $\mathrm{B}$, cytochalasin $\mathrm{D}$, paraformaldehyde (PFA), sivelestat, NE, the NE substrate (MeOSuc)-AAPV(pNA), and thrombin were purchased from Sigma-Aldrich. Additional reagents were as follows: TOPRO-3, phalloidin, SYTO Green (cell-permeable DNA stain), and SYTOX Orange (cell-impermeable DNA stain) (Molecular Probes); Cl-amidine (Calbiochem); DNase (Promega); anti-CD15-microbeads (Miltenyi Biotec); Medium-199 (Lonza), and micrococcal DNase (Worthington).

$n N I F$ and NRP synthesis. nNIF, NRPs, and their specific scrambled peptide controls (Supplemental Table 2) were synthesized by the DNA/Peptide Facility, a unit of the Health Sciences Center Cores at the University of Utah. The core facility also verified the sequence and purity of the provided peptides.

$P M N$ and platelet isolation. PMNs were isolated from ACD or EDTA anticoagulated venous blood from healthy adults, healthy term infants, and prematurely born infants $(28,30)$. For the 8 prematurely born infants from whom we collected cord and peripheral blood samples, cord and peripheral blood plasma and PMN preparations were obtained at 5 separate time points throughout the first 2 months of life. PMN suspensions ( $>96 \%$ pure) were prepared by positive immunoselection using anti-CD15-coated microbeads and an auto-MACS cell sorter (Miltenyi Biotec) and were resuspended at $2 \times 10^{6}$ cells $/ \mathrm{ml}$ concentration in serum-free $\mathrm{M}-199$ media at $37^{\circ} \mathrm{C}$ in $5 \% \mathrm{CO}_{2} / 95 \%$ air. Human platelets were isolated as described (72).

Live cell imaging of NET formation. Qualitative assessment of NET formation was performed as previously reported $(28,30)$. Briefly, primary PMNs isolated from preterm infants, healthy term infants, and healthy adults $\left(2 \times 10^{6}\right.$ cells $\left./ \mathrm{ml}\right)$ were incubated with control buffer or stimulated with indicated agonists or bacteria for 1 hour at $37^{\circ} \mathrm{C}$ in $5 \% \mathrm{CO}_{2} / 95 \%$ air on glass coverslips coated with poly-L-lysine. For selected experiments, primary PMNs were preincubated with autologous plasma, cord blood plasma, nNIF (0.2-70 nM), CRISPP (0.2-70 nM), nNIF-SCR ( $1 \mathrm{nM})$, or CRISPP-SCR (1 nM) for 1 hour prior to stimulation. After preincubation and/or stimulation, PMNs were gently washed with PBS and incubated with a mixture of cell-permeable (SYTO Green, Molecular Probes) and -impermeable (SYTOX Orange, Molecular Probes) DNA fluorescent dyes. Confocal microscopy was accomplished using a FV1000 1X81 confocal microscope and FluoView software (Olympus). Both $20 \times$ and $60 \times$ objectives were used. Z-series images were obtained at a step size $1 \mu \mathrm{m}$ over a range of $20 \mu \mathrm{m}$ for each field. Olympus FluoView and Adobe Photoshop CS2 software were used for image processing.

Imaging of dengue virus-induced NET formation. Using BSL 2 safety protocols, primary PMNs isolated from healthy adults $\left(2 \times 10^{6}\right.$ cells $\left./ \mathrm{ml}\right)$ were incubated with mock infection buffer or live dengue virus (MOI 0.05) as for live cell imaging. After a 1-hour incubation, the infected PMNs were fixed with 2\% PFA for 10 minutes prior to incubation with fluorescently labeled, cell-permeable, and cell-impermeable DNA dyes and imaged as for live cell imaging using confocal microscopy. 
A Peritoneal fluid

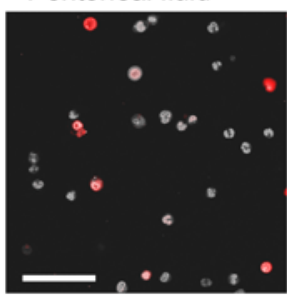

CRISPP

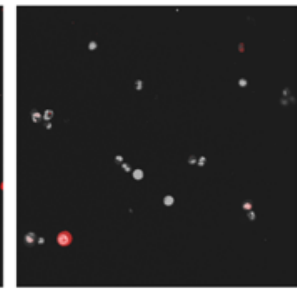

nNIF

CRISPP-SCR

B Peritoneal membrane

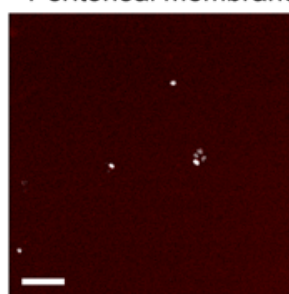

CRISPP

C
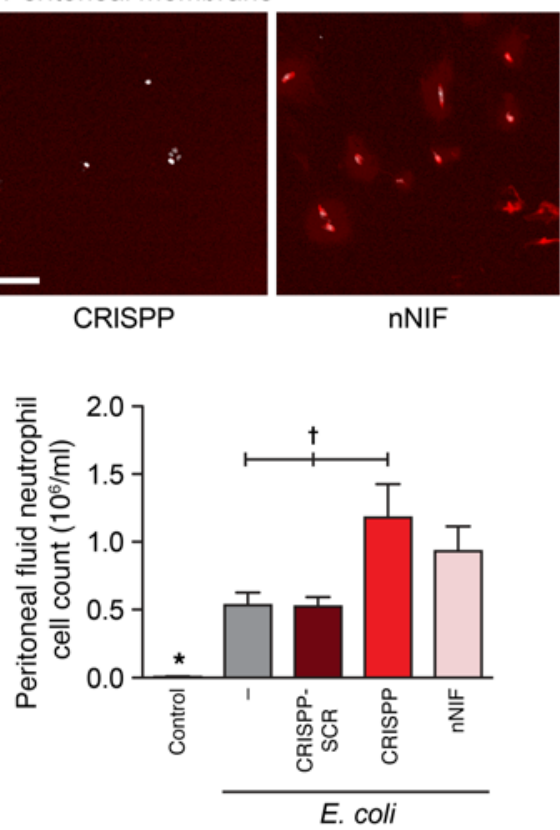

nNIF

D

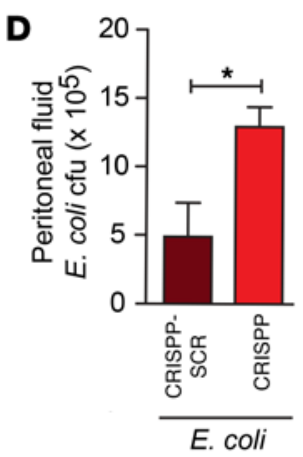

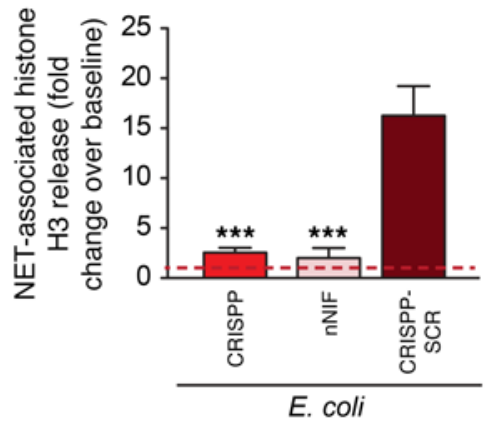

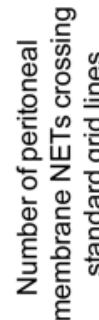

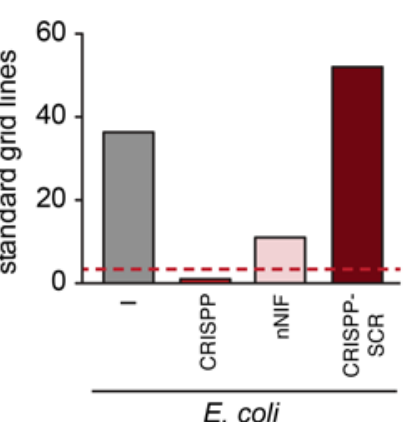

E Peritoneal fluid

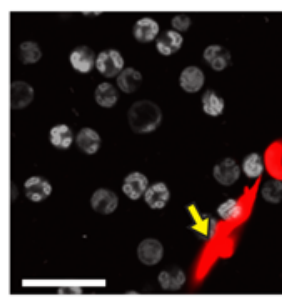

CRISPP

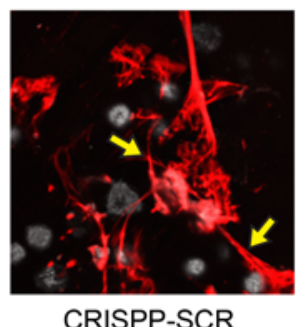

CRISPP-SCR

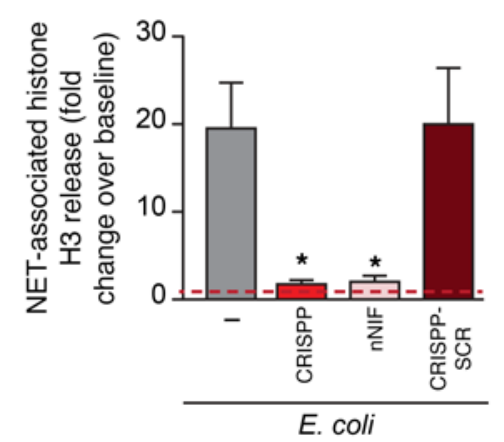

F Peritoneal membrane

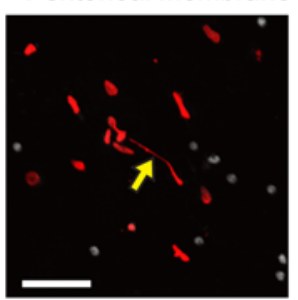

CRISPP

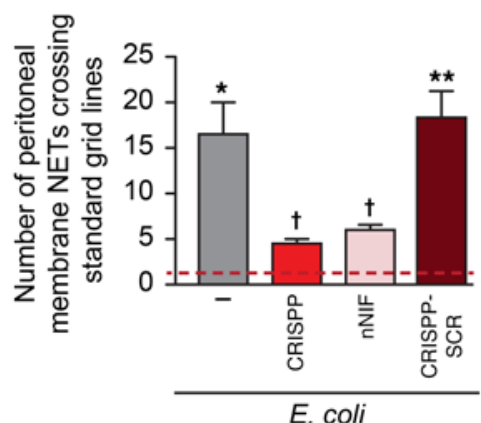

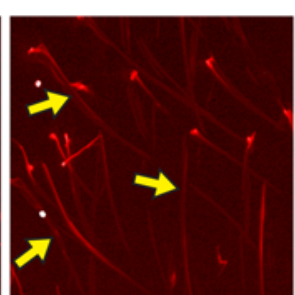

CRISPP-SCR

E. coli 
Figure 8. nNIF and CRISPP inhibit in vivo NET formation. (A and $B$ ) C57BL/ 6 mice not pretreated or pretreated with nNIF, CRISPP, or CRISPPSCR $(10 \mathrm{mg} / \mathrm{kg}$ i.p.; 1 hour $)$ were inoculated with $E$. coli $\left(4.5 \times 10^{7}\right.$ bacteria i.p.). After 3 hours, animals were sacrificed and peritoneal fluid and membranes collected for analysis. (A) Peritoneal fluid NET formation (red fluorescence, yellow arrow) assessed by live cell imaging and histone $\mathrm{H} 3$ release (red dashed line, baseline arbitrarily set at 1 ). Three mice per group. Original magnification, $\times 60$. Scale bars: $50 \mu \mathrm{m}$. One-way ANOVA with Tukey's post hoc testing. ${ }^{* *} P<0.001$, CRISPP and nNIF versus CRISPPSCR. (B) NET formation on the surfaces of peritoneal membranes (red fluorescence, yellow arrows) was quantified by counting the number of NETs that crossed standardized grid lines in 4 random microscopic fields using Image) software. Original magnification, $\times 60$. Scale bars: $50 \mu \mathrm{m}$. A second experiment yielded a similar pattern. (C) C57BL/6 mice not pretreated (left 2 bars) or pretreated with CRISPP, nNIF, or CRISPP-SCR were inoculated with $E$. coli i.p. as in $\mathbf{A}$ and $\mathbf{B}$, and neutrophil numbers in peritoneal fluid were counted after 3 hours (3-5 mice/group). One-way ANOVA with Newman-Keul post hoc testing; ${ }^{\dagger} P<0.05$, CRISPP vs. CRISPP-SCR or not pretreated; ${ }^{*} P<0.05$, control vs. all other groups. (D) C57BL/ 6 mice were pretreated with CRISPP or CRISPP-SCR and inoculated with $E$. coli as in $\mathbf{A}$ and $\mathbf{B}$ (5 animals/group). After 3 hours, bacteria CFU in the peritoneal fluid were measured. Single-tailed Mann-Whitney $U$ test, ${ }^{*} P<0.05$. (E and F) Swiss Webster mice not pretreated or pretreated with nNIF, CRISPP, or CRISPP-SCR were inoculated with E. coli i.p. as in A and B. After 3 hours, peritoneal fluid and membranes were collected. (E) Peritoneal fluid NET formation, imaged and measured as in $\mathbf{A}$ (10 mice/group). ${ }^{*} P<0.05$, CRISPP/E. coli and nNIF/E. coli compared with CRISPP-SCR/E. coli and E. coli. (F) NET formation on peritoneal membrane surfaces, imaged and quantitated as in $\mathbf{B}$ (3 mice in each group). ${ }^{*} P<0.05, E$. coli versus control (red dashed line); ${ }^{*} P<0.01$, CRISPP-SCR/E. coli versus control; ${ }^{+} P<0.05$, CRISPP/E. coli and nNIF/E. coli versus CRISPP-SCR/E. coli. Oneway ANOVA with Tukey's post-hoc testing applied in $\mathbf{E}$ and $\mathbf{F}$.

Quantitation of NET formation - NET-associated histone H3 content. We determined NET-associated histone $\mathrm{H} 3$ content as previously described (30). After live cell imaging of control and stimulated primary PMNs $\left(2 \times 10^{6} / \mathrm{ml}\right.$; various agonists $)$, the cells were incubated with PBS containing DNase $(40 \mathrm{U} / \mathrm{ml})$ for 15 minutes at room temperature to break down and release NETs formed in response to stimulation. The supernatant was gently removed and centrifuged at $420 \mathrm{~g}$ for $5 \mathrm{~min}$ utes. The cell-free supernatant was then mixed 3:1 with $4 \times$ Laemmli buffer prior to Western blotting. We used a polyclonal primary antibody against human histone $\mathrm{H} 3$ protein (Cell Signaling) and infrared-conjugated secondary antibodies (LI-COR). Imaging and densitometry were performed on the Odyssey infrared imaging system (LI-COR). We previously validated this assay as a surrogate for NET formation under in vitro conditions (30) as employed in the current studies.

Isolation and identification of nNIF in umbilical cord blood plasma. Two plasma samples from a single preterm infant, one from the umbilical cord blood and one from a peripheral blood sample taken on ex utero day 28, were subjected to abundant plasma protein removal (Norgen) prior to $2 \mathrm{D}$ electrophoresis using separation first by isoelectric focusing (pH range 3-8) and then by size (TGX Precast Gel, Bio-Rad). The resulting gels were compared for differential protein content. Six differentially expressed protein clusters ("spots") were sent to the University of Utah Proteomics Core for analysis. Following trypsin digestion and tandem mass spectroscopy using an LTQ-FT ion-trap/FTMS hybrid mass spectrometer (ThermoElectron), candidate proteins/peptides were identified as potential NET-inhibitory factors.

Affinity removal of $n N I F$. Plasma samples were subjected to abundant plasma protein removal (Norgen). A polyclonal antibody raised against the carboxy terminal 18 amino acids A1AT (LifeSpan BioSciences) coupled to resin beads from an immunoprecipitation kit purchased from Thermo Fisher Scientific was then used to immunodeplete the samples. Nonimmune IgG coupled to resin beads was used in parallel as a control. Plasma was diluted in lysis buffer from the kit and incubated with the anti-A1AT C terminus antibody-coupled beads or with control beads overnight at $4^{\circ} \mathrm{C}$. The beads were then separated by centrifugation and the immunodepleted and control plasma samples collected. The A1AT C terminus antibody coupled and control beads were resuspended in kit-included elution buffer for 10 minutes at room temperature, followed by centrifugation and collection of the eluate and control supernatants. The eluate was analyzed by Western blotting (16.5\% Tris-Tricine Gel, Bio-Rad) using the A1AT C terminus antibody and by tandem mass spectroscopy. Immunodepleted plasma and eluate samples were examined in assays of NET formation. Active full-length native and recombinant A1AT (both from Sigma-Aldrich) were suspended in elution buffer and tested in parallel.

Bacterial killing assay. We determined NET-mediated and phagocytic bacterial killing by primary human PMNs as previously described (28).

Chemotaxis assay. Chemotaxis by PMNs isolated from healthy adult donors was assessed using a modified Boyden chamber assay with or without a 1-hour preincubation with nNIF (1 nM), CRISPP (1 nM), or CRISPP-SCR (1 nM). Recombinant human IL-8 (2 ng/ml) was used as the chemoattractant. Chemotaxis through a 5 -micron filter was determined by counting PMNs in 10 randomly selected high-power fields as previously described (73). In separate experiments, nNIF, CRISPP, or CRISPP-SCR (all at $1 \mathrm{nM}$ ) were evaluated for chemoattractant activity using the same system.

Phagocytosis assay. PMNs were isolated from blood of healthy adult donors and resuspended in M-199 at a concentration of $2 \times 10^{6}$ cells/ ml. Leukocytes were preincubated for 60 minutes under standard conditions with cytochalasin D and B $(10 \mu \mathrm{M})$, nNIF (1 nM), CRISPP $(1 \mathrm{nM})$, or CRISPP-SCR $(1 \mathrm{nM})$. Following preincubation, PMNs were incubated with $6 \times 10^{6} \mathrm{E}$. coli bioparticles (Molecular Probes) on a rotator for 4 hours at $37^{\circ} \mathrm{C}$ in $5 \% \mathrm{CO}_{2} / 95 \%$ air. The PMNs were then washed and resuspended in the starting volume of M-199 before being spun down onto glass coverslips and fixed with 2\% PFA for 10 minutes and permeabilized with $0.1 \%$ Triton $\mathrm{X}-100$ for 10 minutes. Leukocytes were stained with WGA 555 (Invitrogen) and TOPRO-3 (Molecular Probes), and randomly selected high-power visual field images were captured using confocal microscopy. We used ImageJ software (NIH) to determine the percentage of PMNs that were positive for fluorescently labeled E. coli bioparticles detected at $488 \mathrm{~nm}$.

Reactive oxygen species generation. Human PMNs isolated from healthy adult whole blood were resuspended to a concentration of $2 \times 10^{6}$ cells $/ \mathrm{ml}$ in M-199 media and preincubated with or without CRISPP $(1 \mathrm{nM})$ or CRISPP-SCR $(1 \mathrm{nM})$ peptide for 1 hour at $37^{\circ} \mathrm{C}$ in $5 \% \mathrm{CO}_{2} / 95 \%$ air. The PMNs were then stimulated with LPS (100 ng/ $\mathrm{ml}$ ) for 1 hour, washed, and resuspended with a dihydrorhodamine (7.25mM; Molecular Probes) and catalase (1000 units/ml; SigmaAldrich) mixture and incubated at $37^{\circ} \mathrm{C}$ for 10 minutes. After incubation, samples were placed at $4^{\circ} \mathrm{C}$ and analyzed for ROS-dependent fluorescence using flow cytometry as performed in the University of Utah core facility (BD, CellQuest software).

Platelet activation assays. P-selectin translocation and surface display by activated platelets (74) and formation of platelet-neutrophil aggregates (75) were measured as described. 

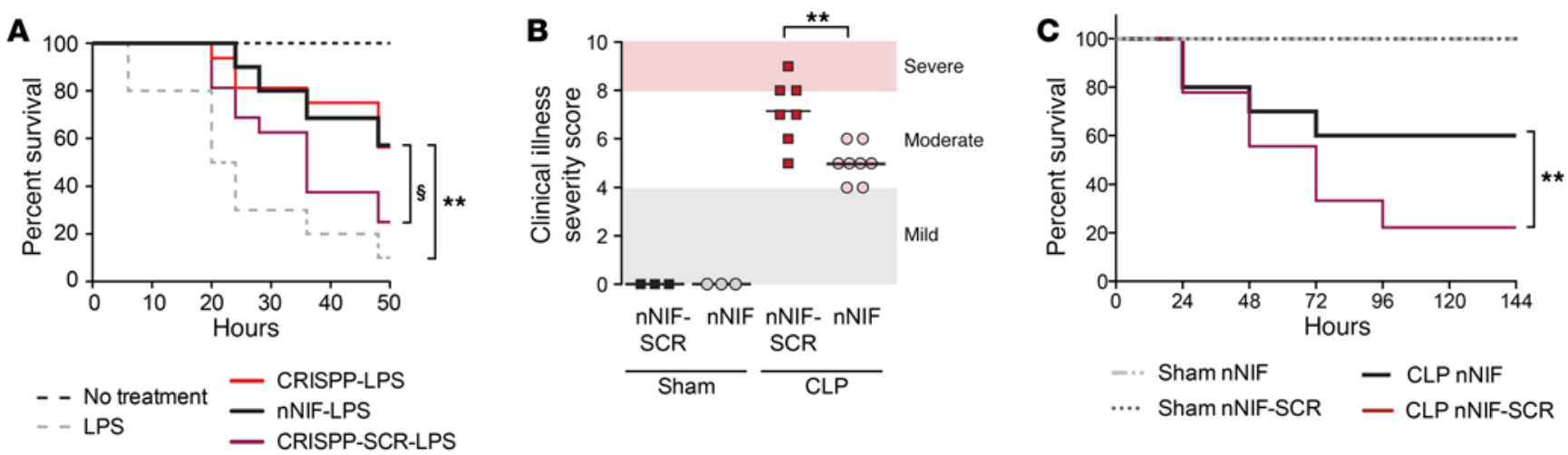

Figure 9. nNIF and CRISPP improve survival in experimental systemic inflammation. (A) C57BL/6 mice were challenged with LPS (25 mg/kg i.p.). CRISPP, $\mathrm{nNIF}$, or CRISPP-SCR (10 mg/kg i.p.) was given 1 hour before and 6 hours after LPS. Animals with no treatments or given LPS alone were studied in parallel ( $n \geq 10$ mice for each condition). ${ }^{* *} P<0.01$, log-rank (Mantel-Cox) statistical tool used. The survival difference between nNIF-LPS and CRISPP-LPS compared with CRISPP-SCR-LPS trended toward significance. ${ }^{\$} P=0.051$. (B) C57BL/6 mice were subjected to CLP. nNIF or nNIF-SCR (10 mg/kg i.p.) was given 1 hour before and 6 hours after surgery ( $n \geq 7$ in each group). Mice subjected to sham surgery were studied in parallel ( $n=3$ in each group). Clinical illness severity scores (52) were determined at 24 hours. One-way ANOVA with Newman-Keul post hoc testing; ${ }^{* *} P<0.01$, nNIF vs. nNIF-SCR groups. (C) Mice assessed for severity of systemic illness in B were then followed daily and survivors sacrificed at 144 hours. Log-rank (Mantel-Cox) statistical tool used. ${ }^{* *} P<0.01$.

Nuclear decondensation assay. PMNs were isolated and resuspended to $2 \times 10^{6}$ cells $/ \mathrm{ml}$ in M-199 media, preincubated with $\mathrm{nNIF}(1 \mathrm{nM})$, CRISPP (1 nM), nNIF-SCR ( $1 \mathrm{nM})$, CRISPP-SCR ( $1 \mathrm{nM})$, or the PAD4 inhibitor Cl-amidine $(10 \mu \mathrm{M})$ for 1 hour at $37^{\circ} \mathrm{C}$ in $5 \% \mathrm{CO}_{2} / 95 \%$ air, and treated with or without PMA $(20 \mathrm{nM})$ on poly-L-lysine-coated glass coverslips for 2 hours. Nuclear decondensation was identified as described (6). Five randomly selected high-power visual fields per sample were captured via confocal microscopy and analyzed for nuclear area using the cell-permeable, fluorescent DNA dye SYTO Green. The nuclear pixel areas of more than 100 individual cells per high-power field were determined using Image J software (NIH).

PAD4 activity assay. We determined nNIF inhibition of PAD4 activity using a PAD4 Inhibitor Screening Assay Kit (Cayman). Briefly, nNIF $(1 \mathrm{nM})$ was incubated with recombinant PAD4 and PAD4 enzyme substrate $(2 \mathrm{mM})$ in PAD4 assay reagent for 30 minutes at $37^{\circ} \mathrm{C}$. The PAD4 inhibitor $\mathrm{Cl}$-amidine $(10 \mu \mathrm{M})$ was used as a positive control for PAD4 inhibition. The reaction was stopped with PAD4 Stop Solution and detected using an included ammonia detector assay. Ammonia detector fluorescence was measured at $470 \mathrm{~nm}$ following excitation at $405 \mathrm{~nm}$ on a SpectraMax M5 fluorescence plate reader (Molecular Devices).

Histone $\mathrm{H} 3$ citrullination determination. Adult PMNs were stimulated with PMA (20 nM) for 15 minutes at $37^{\circ} \mathrm{C}$ in $5 \% \mathrm{CO}_{2} / 95 \%$ air following a 15 -minute preincubation with nNIF, CRISPP, nNIF-SCR, or CRISPP-SCR $(1 \mathrm{nM})$ or with Cl-amidine $(10 \mu \mathrm{M})$, spun onto poly-Llysine-coated slides, and examined by immunocytochemistry with a primary antibody used to detect human citrullinated histone $\mathrm{H} 3$ (Abcam). Imaging was accomplished via confocal microscopy using a FV1000 1X81 confocal microscope and FluoView software (Olympus). Semiquantitation was accomplished using ImageJ software (NIH) to determine the average citrullinated histone $\mathrm{H} 3$ content per cell.

CRISPP peptide cellular localization. FLAG-tagged CRISPP (F-CRISPP) and FLAG-tagged CRISPP-SCR (F-CRISPP-SCR) peptides were synthesized by our core facility and detected using immunocytochemistry. Adult neutrophils were preincubated with either F-CRISPP $(1 \mathrm{nM})$ or F-CRISPP-SCR $(1 \mathrm{nM})$ for 1 hour at $37^{\circ} \mathrm{C}$ in $5 \% \mathrm{CO}_{2} / 95 \%$ air, followed by stimulation with LPS $(100 \mathrm{ng} / \mathrm{ml})$ for 2 hours. The
PMNs were then spun down onto glass coverslips with 2\% PFA fixation and $0.1 \%$ Triton X-100 permeabilization. FLAG-tagged peptide was detected using a monoclonal anti-FLAG antibody (Sigma-Aldrich) with TOPRO-3 as a nuclear counterstain.

Mouse models of E. coli and LPS-induced peritonitis. C57BL/6 or Swiss Webster mice were pretreated in blinded fashion with CRISPP (10 mg/ $\mathrm{kg})$, nNIF (10 mg/kg), or CRISPP-SCR $(10 \mathrm{mg} / \mathrm{kg})$ by i.p. injection 1 hour prior to infection (E. coli, $4.5 \times 10^{7} \mathrm{CFU} /$ mouse, i.p.) or inoculation (LPS, $25 \mathrm{mg} / \mathrm{kg}$, i.p.) (Sigma-Aldrich). Control mice were injected with saline alone. The mice were sacrificed in a $\mathrm{CO}_{2}$ chamber 3 hours after infection/ inoculation and the peritoneal fluid and membrane samples harvested. Briefly, the abdomen was disinfected and opened in the midline without injuring the muscle. The peritoneal cavity was lavaged with sterile saline solution $(1 \mathrm{ml})$ and analyzed for in vivo NET formation, bacteriology, and leukocyte accumulation. NETs in the peritoneal fluid were qualitatively and quantitatively analyzed using live cell imaging with confocal microscopy and NET-associated histone $\mathrm{H} 3$ release assays. NETs on the serosal surface of the peritoneal membrane were assessed quantitatively using live cell imaging, followed by standardized grid analysis of 5 randomly selected high-power visual fields per tissue sample (ImageJ, NIH). We quantified peritoneal bacterial CFU counts by permeabilizing all recovered leukocytes with $0.1 \%$ Triton $\mathrm{X}-100$ for 10 minutes and performing serial dilutions and bacterial cultures on $5 \%$ sheep blood agar plates (Hardy Diagnostics). After a 24-hour incubation, bacterial counts were determined. Total leukocyte counts in the peritoneal lavage were determined in Neubauer chambers using an optical microscope after dilution in Turk's solution (2\% acetic acid). Differential leukocyte analysis was performed using a $60 \times$ oil immersion objective to assess morphology of cytocentrifuged cells stained with May-Gruenwald-Giemsa dye. All mice were included in the final analysis.

Mouse model of systemic inflammation induced by LPS (endotoxemia). C57BL/6 mice were pretreated in blinded fashion with CRISPP (10 mg/kg), nNIF (10 mg/kg), or CRISPP-SCR (10 mg/kg) by i.p. injection 1 hour prior to and 6 hours after inoculation with LPS $(25 \mathrm{mg} / \mathrm{kg}$, i.p. injection). Control mice were injected i.p. with saline alone. Fluid resuscitation and antibiotic treatment were not used in these experi- 
ments. Survival was assessed over 50- or 72-hour intervals. All mice were included in the final survival analysis.

Mouse model of polymicrobial sepsis using CLP. C57BL/6 mice were anesthetized with ketamine/xylazine $(100 \mathrm{mg} / \mathrm{kg}$ and $10 \mathrm{mg} / \mathrm{kg}$, i.p., respectively), and CLP was performed as previously described (52). nNIF $(10 \mathrm{mg} / \mathrm{kg})$ or nNIF-SCR $(10 \mathrm{mg} / \mathrm{kg})$ i.p. was given 1 hour prior to and 6 hours after CLP surgery. The animals received subcutaneous sterile isotonic saline $(1 \mathrm{ml})$ for fluid resuscitation immediately after the surgery. Sham-operated mice were subjected to identical procedures except that CLP was not done. Twenty-four hours after CLP, all animals were scored for clinical illness severity as previously described (52). In this assessment, higher scores reflect increased illness severity. Survival of mice in the nNIF/CLP $(n=7)$, nNIF-SCR/ CLP $(n=8)$, nNIF/sham surgery $(n=3)$, or nNIF-SCR/sham surgery $(n=3)$ groups was followed for 5 days after the surgical procedure.

$N E$ activity assay. Synthetic fluorogenic substrate of $\mathrm{NE}$ (MeOSuc)AAPV-(pNA) $(160 \mu \mathrm{M})$ was incubated with bioactive NE (500 nM) with or without the NE inhibitor sivelestat $(160 \mu \mathrm{M})$ or nNIF $(50 \mathrm{nM})$ for 3 hours at $37^{\circ} \mathrm{C}$. The reactions were quenched with $5 \%$ glacial acetic acid and centrifuged at 18,500 RCF for 5 minutes. Chromatograms were obtained using an Agilent 1100 Series HPLC and a Phenomenex $5 \mu \mathrm{m}$ C18 Luna column (100 ̊, $4.6 \times 150 \mathrm{~mm}$ ) over a 30-minute $10 \%$ to $90 \%$ B gradient (buffer A $0.1 \%$ TFA in $\mathrm{H}_{2} \mathrm{O}$, buffer B $0.1 \%$ TFA in ACN). Mass spectra were obtained for secondary validation of the reaction products using an API 3500 triple quadrupole mass spectrometer. Chromatograms were offset on both the $x$ and $y$ axes (by 0.5 minutes and $0.1 \mathrm{~A}_{214}$, respectively) for greater visibility. Relative $\mathrm{A}_{214}$ was determined by normalizing all of the data to the tallest HPLC peak displayed in each graph.

Statistics. We used GraphPad Prism statistical software (version 5) to analyze results. Mean \pm SEM was determined for each experimental variable. All data used in each statistical test met the assumption of the specific test and were normally distributed. $P<0.05$ was considered statistically significant.
Study approval. The University of Utah Institutional Review Board approved this study (IRB nos. 0392, 11919, and 39621), and all human subjects provided informed consent in accordance with the Declaration of Helsinki. All murine experiments performed were approved by the University of Utah Institutional Animal Care and Use Committee (no. 12-11017) in a facility approved by the American Association of Laboratory Animal Care.

\section{Author contributions}

CCY, ASW, and GAZ conceived of the project and designed the experiments. CCY and CLK performed patient phenotyping. MTR provided statistical expertise. MJC, HS, JAW, RAC, AVDA, CVA, SS, and JMF conducted experiments. RAC, AVDA, CVA, ESH, and JWR developed experimental tools or mouse models. CCY and GAZ wrote the manuscript.

\section{Acknowledgments}

We thank Diana Lim, Alex Greer, and Kendra Richardson for graphics assistance and manuscript preparation, Heather Major for discussions, Neal Tolley for technical assistance, and Michael Kay and John Hoidal for invaluable help with critical enzyme assays. This work was supported in part by the US NIH (K08HD049699 to CCY; K01GM103806 to JWR; R01HL126547 and R01AG048022 to MTR, R37HL044525 to GAZ; R01HL066277 to ASW; U54HL112311 to MTR, ASW, and GAZ), and the Volkswagen Foundation (Lichtenberg Professorship to HS).

Address correspondence to: Christian Con Yost, Pediatrics, University of Utah School of Medicine, Williams Building, 295 Chipeta Way, Salt Lake City, Utah 84108, USA. Phone: 801.581.7052; E-mail: christian.yost@u2m2.utah.edu.

MTR's present address is: Geriatric Research \& Education Center (GRECC) George E. Wahlen VAMC, Salt Lake City, Utah, USA.
1. Sørensen OE, Borregaard N. Neutrophil extracellular traps - the dark side of neutrophils. JClin Invest. 2016;126(5):1612-1620.

2. Brinkmann V, Zychlinsky A. Neutrophil extracellular traps: is immunity the second function of chromatin?. JCell Biol. 2012;198(5):773-783.

3. Yipp BG, Kubes P. NETosis: how vital is it? Blood. 2013;122(16):2784-2794.

4. Brinkmann V, et al. Neutrophil extracellular traps kill bacteria. Science. 2004;303(5663):1532-1535.

5. Yipp BG, et al. Infection-induced NETosis is a dynamic process involving neutrophil multitasking in vivo. Nat Med. 2012;18(9):1386-1393.

6. Papayannopoulos V, Metzler KD, Hakkim A, Zychlinsky A. Neutrophil elastase and myeloperoxidase regulate the formation of neutrophil extracellular traps. J Cell Biol. 2010;191(3):677-691.

7. Wang Y, et al. Histone hypercitrullination mediates chromatin decondensation and neutrophil extracellular trap formation. J Cell Biol. 2009;184(2):205-213.

8. Li P, Li M, Lindberg MR, Kennett MJ, Xiong $\mathrm{N}$, Wang Y. PAD4 is essential for antibacterial innate immunity mediated by neutrophil extracellular traps. J Exp Med. 2010;207(9):1853-1862.
9. Kolaczkowska E, et al. Molecular mechanisms of NET formation and degradation revealed by intravital imaging in the liver vasculature. Nat Commun. 2015;6:6673.

10. Nauseef WM. How human neutrophils kill and degrade microbes: an integrated view. Immunol Rev. 2007;219:88-102.

11. Bianchi M, et al. Restoration of NET formation by gene therapy in CGD controls aspergillosis. Blood. 2009;114(13):2619-2622.

12. $\mathrm{Xu}$ J, et al. Extracellular histones are major mediators of death in sepsis. Nat Med. 2009;15(11):1318-1321.

13. Clark SR, et al. Platelet TLR4 activates neutrophil extracellular traps to ensnare bacteria in septic blood. Nat Med. 2007;13(4):463-469.

14. McDonald B, Urrutia R, Yipp BG, Jenne CN, Kubes P. Intravascular neutrophil extracellular traps capture bacteria from the bloodstream during sepsis. Cell Host Microbe. 2012;12(3):324-333.

15. Caudrillier A, et al. Platelets induce neutrophil extracellular traps in transfusion-related acute lung injury. J Clin Invest. 2012;122(7):2661-2671.

16. Sayah DM, et al. Neutrophil extracellular traps are pathogenic in primary graft dysfunction after lung transplantation. Am J Respir Crit Care Med. 2015;191(4):455-463.

17. Chen G, Zhang D, Fuchs TA, Manwani D, Wagner DD, Frenette PS. Heme-induced neutrophil extracellular traps contribute to the pathogenesis of sickle cell disease. Blood. 2014;123(24):3818-3827.

18. Lood C, et al. Neutrophil extracellular traps enriched in oxidized mitochondrial DNA are interferogenic and contribute to lupus-like disease. Nat Med. 2016;22(2):146-153.

19. Fuchs TA, et al. Extracellular DNA traps promote thrombosis. Proc Natl Acad Sci U S A. 2010;107(36):15880-15885.

20. Pillai PS, et al. Mx1 reveals innate pathways to antiviral resistance and lethal influenza disease. Science. 2016;352(6284):463-466.

21. McDonagh S, Maidji E, Ma W, Chang HT, Fisher S, Pereira L. Viral and bacterial pathogens at the maternal-fetal interface. J Infect Dis. 2004;190(4):826-834.

22. Palmer C, Bik EM, DiGiulio DB, Relman DA, Brown PO. Development of the human infant intestinal microbiota. PLoS Biol. 2007;5(7):e177.

23. Jost T, Lacroix C, Braegger CP, Chassard C. New insights in gut microbiota establishment 
in healthy breast fed neonates. PLoS One. 2012;7(8):e44595.

24. Deshmukh HS, et al. The microbiota regulates neutrophil homeostasis and host resistance to Escherichia coli K1 sepsis in neonatal mice. Nat Med. 2014;20(5):524-530.

25. Dowling DJ, Levy O. Ontogeny of early life immunity. Trends Immunol. 2014;35(7):299-310.

26. Adkins B. Neonatal immunology: responses to pathogenic microorganisms and epigenetics reveal an "immunodiverse" developmental state. Immunol Res. 2013;57(1-3):246-257.

27. Elahi S, et al. Immunosuppressive CD71+ erythroid cells compromise neonatal host defence against infection. Nature. 2013;504(7478):158-162.

28. Yost CC, et al. Impaired neutrophil extracellular trap (NET) formation: a novel innate immune deficiency of human neonates. Blood. 2009;113(25):6419-6427.

29. Marcos V, et al. Delayed but functional neutrophil extracellular trap formation in neonates. Blood. 2009;114(23):4908-4911; author reply 4911.

30. McInturff AM, et al. Mammalian target of rapamycin regulates neutrophil extracellular trap formation via induction of hypoxia-inducible factor 1 a. Blood. 2012;120(15):3118-3125.

31. Janciauskiene SM, Bals R, Koczulla R, Vogelmeier C, Köhnlein T, Welte T. The discovery of a1-antitrypsin and its role in health and disease. Respir Med. 2011;105(8):1129-1139.

32. Jonigk $\mathrm{D}$, et al. Anti-inflammatory and immunomodulatory properties of $\alpha 1$-antitrypsin without inhibition of elastase. Proc Natl Acad Sci U S A. 2013;110(37):15007-15012.

33. Farley K, Stolley JM, Zhao P, Cooley J, Remold-O'Donnell E. A serpinB1 regulatory mechanism is essential for restricting neutrophil extracellular trap generation. JImmunol. 2012;189(9):4574-4581.

34. Frenzel E, et al. Does augmentation with alpha1antitrypsin affect neutrophil extracellular traps formation? Int J Biol Sci. 2012;8(7):1023-1025.

35. Cercek L, Cercek B. Cancer-associated SCM-recognition, immunedefense suppression, and serine protease protection peptide. Part I. Isolation, amino acid sequence, homology, and origin. Cancer Detect Prev. 1992;16(5-6):305-319.

36. Cercek L, Cercek B. Cancer-associated SCM-recognition, immunedefense suppression, and serine protease protection peptide. Part II. Immunedefense suppressive effects of the CRISPPs peptide. Cancer Detect Prev. 1993;17(3):433-445.

37. Cercek L, Cercek B. Cancer-associated SCM-recognition, immune defense suppression, and serine protease protection peptide. Part III. CRISPP peptide protection of serine proteases against inhibition. Cancer Detect Prev. 1993;17(3):447-454.

38. Saffarzadeh M, Preissner KT. Fighting against the dark side of neutrophil extracellular traps in disease: manoeuvres for host protection. Curr Opin Hematol. 2013;20(1):3-9.

39. Niemann MA, Narkates AJ, Miller EJ. Isolation and serine protease inhibitory activity of the 44-residue, C-terminal fragment of alpha 1-antitrypsin from human placenta. Matrix. 1992;12(3):233-241.

40. Niemann MA, Baggott JE, Miller EJ. Binding of SPAAT, the 44-residue C-terminal peptide of alpha 1-antitrypsin, to proteins of the extracellular matrix. JCell Biochem. 1997;66(3):346-357.

41. Fuchs TA, et al. Novel cell death program leads to neutrophil extracellular traps. JCell Biol. 2007;176(2):231-241.

42. Pilsczek FH, et al. A novel mechanism of rapid nuclear neutrophil extracellular trap formation in response to Staphylococcus aureus. J Immunol. 2010;185(12):7413-7425.

43. Saitoh T, et al. Neutrophil extracellular traps mediate a host defense response to human immunodeficiency virus-1. Cell Host Microbe. 2012;12(1):109-116

44. Jenne CN, et al. Neutrophils recruited to sites of infection protect from virus challenge by releasing neutrophil extracellular traps. Cell Host Microbe. 2013;13(2):169-180.

45. Raftery MJ, et al. $\beta 2$ integrin mediates hantavirusinduced release of neutrophil extracellular traps. JExp Med. 2014;211(7):1485-1497.

46. Cheung R, et al. Activation of MDL-1 (CLEC5A) on immature myeloid cells triggers lethal shock in mice. J Clin Invest. 2011;121(11):4446-4461.

47. Gladwin MT, Ofori-Acquah SF. Erythroid DAMPs drive inflammation in SCD. Blood. 2014;123(24):3689-3690.

48. Vieira-de-Abreu A, Campbell RA, Weyrich AS, Zimmerman GA. Platelets: versatile effector cells in hemostasis, inflammation, and the immune continuum. Semin Immunopathol. 2012;34(1):5-30.

49. Branzk N, Papayannopoulos V. Molecular mechanisms regulating NETosis in infection and disease. Semin Immunopathol. 2013;35(4):513-530.

50. Tanaka $\mathrm{K}$, et al. In vivo characterization of neutrophil extracellular traps in various organs of a murine sepsis model. PLOS ONE. 2014;9(11):e111888.

51. Hubbard WJ, et al. Cecal ligation and puncture. Shock. 2005;24(Suppl 1):52-57.

52. Araújo CV, et al. A PPAR $\gamma$ agonist enhances bacterial clearance through neutrophil extracellular trap formation and improves survival in sepsis. Shock. 2016;45(4):393-403.

53. Czaikoski PG, et al. Neutrophil extracellular traps induce organ damage during experimental and clinical Sepsis. PLoS One. 2016;11(2):e0148142.

54. Nathan C. Points of control in inflammation. Nature. 2002;420(6917):846-852.

55. Medzhitov R. Origin and physiological roles of inflammation. Nature. 2008;454(7203):428-435

56. Arck PC, Hecher K. Fetomaternal immune crosstalk and its consequences for maternal and offspring's health. Nat Med. 2013;19(5):548-556.

57. Levy O. Innate immunity of the newborn: basic mechanisms and clinical correlates. Nat Rev Immunol. 2007;7(5):379-390.

58. Gupta AK, Hasler P, Holzgreve W, Gebhardt S, Hahn S. Induction of neutrophil extracellular DNA lattices by placental microparticles and IL-8 and their presence in preeclampsia. Hum Immunol. 2005;66(11):1146-1154.
59. Sangaletti S, et al. Neutrophil extracellular traps mediate transfer of cytoplasmic neutrophil antigens to myeloid dendritic cells toward ANCA induction and associated autoimmunity. Blood. 2012;120(15):3007-3018.

60. Castellucci M, Theelen T, Pompili E, Fumagalli L, De Renzis G, Mühlhauser J. Immunohistochemical localization of serine-protease inhibitors in the human placenta. Cell Tissue Res. 1994;278(2):283-289.

61. Frochaux V, Hildebrand D, Talke A, Linscheid MW, Schlüter H. Alpha-1-antitrypsin: a novel human high temperature requirement protease A1 (HTRA1) substrate in human placental tissue. PLoS One. 2014;9(10):e109483.

62. Niemann MA, Baggott JE, Miller EJ. Inhibition of human serine proteases by SPAAT, the C-terminal 44-residue peptide from alpha1-antitrypsin. Biochim Biophys Acta. 1997;1340(1):123-130.

63. Pei D, Majmudar G, Weiss SJ. Hydrolytic inactivation of a breast carcinoma cell-derived serpin by human stromelysin-3. JBiol Chem. 1994;269(41):25849-25855.

64. Cools-Lartigue J, et al. Neutrophil extracellular traps sequester circulating tumor cells promote metastasis. JClin Invest. 2013;123(8):3446-3458.

65. Byrd AS, et al. NETosis in Neonates: Evidence of a reactive oxygen species-independent pathway in response to fungal challenge. J Infect Dis. 2016;213(4):634-639.

66. Schauer C, et al. Aggregated neutrophil extracellular traps limit inflammation by degrading cytokines and chemokines. Nat Med.2014;20(5):511-517.

67. Zabieglo K, et al. The inhibitory effect of secretory leukocyte protease inhibitor (SLPI) on formation of neutrophil extracellular traps. J Leukoc Biol. 2015;98(1):99-106.

68. Yost CC. Toward the "ideal" inhibitor of NETs. Blood. 2014;123(16):2439-2440.

69. Lewis HD, et al. Inhibition of PAD4 activity is sufficient to disrupt mouse and human NET formation. Nat Chem Biol. 2015;11(3):189-191.

70. Wildhagen KC, et al. Nonanticoagulant heparin prevents histone-mediated cytotoxicity in vitro and improves survival in sepsis. Blood. 2014;123(7):1098-1101.

71. Nathan C. Neutrophils and immunity: challenges and opportunities. Nat Rev Immunol. 2006;6(3):173-182.

72. Weyrich AS, et al. Activated platelets signal chemokine synthesis by human monocytes. JClin Invest. 1996;97(6):1525-1534.

73. Hill HR, et al. Defect in neutrophil granulocyte chemotaxis in Job's syndrome of recurrent "cold" staphylococcal abscesses. Lancet. 1974;2(7881):617-619.

74. van Velzen JF, Laros-van Gorkom BA, Pop GA, van Heerde WL. Multicolor flow cytometry for evaluation of platelet surface antigens and activation markers. Thromb Res. 2012;130(1):92-98.

75. Evangelista V, et al. Platelet/polymorphonuclear leukocyte interaction in dynamic conditions: evidence of adhesion cascade and cross talk between P-selectin and the beta 2 integrin CD11b/CD18. Blood.1996;88(11):4183-4194. 